\title{
Religion and Innovation in Europe: Implications for Product Life-Cycle Management
}

\author{
Almudena Recio-Román ${ }^{1}$, Manuel Recio-Menéndez ${ }^{2, *}$ and María Victoria Román-González ${ }^{2}$ \\ 1 Tilburg University, Professor Cobbenhagenlaan 9 9.14, 5037 DA Tilburg, North Brabant, The Netherlands; \\ a.recioroman@tilburguniversity.edu \\ 2 Departament of Economy and Business, University of Almería, Carretera de Sacramento s.n., \\ 04120 Almería, Spain; mvroman@ual.es \\ * Correspondence: mrecio@ual.es
}

Received: 8 September 2019; Accepted: 12 October 2019; Published: 21 October 2019

check for updates

\begin{abstract}
This paper analyzes the relationship between religion and innovation in Europe. To the best knowledge of the authors, no paper has been published about the association of religion with innovation and innovative products and services, at an individual level, for all the countries that belong to the European Union. This is the main goal of our paper. The results show that the orientation of innovativeness depends on religion. This study contains a segmentation of the main religions in Europe, taking into account their innovative profile. Connecting the characteristics of the religious segments found and the innovations life-cycle concept, companies have a tool to manage different innovations' evolutive stages taking into consideration the religion of their customers. The European policy-makers, still dominated by a traditional innovation approach, gain a demand-side perspective to improve citizen's innovativeness awareness and acceptance. Finally, religiosity does not seem to have a very strong relationship with attitudes towards innovation once we control for religious affiliation.
\end{abstract}

Keywords: innovation; religion; religiosity; Europe; consumer behavior; product life-cycle

\section{Introduction}

In 2010, more than 5.8 million people ( $84 \%$ of total world population) declared themselves religious to some extent. That year, the major religious groups were Christians (32\%), Muslims (23\%), Hindus (15\%), Buddhists (7\%) and Jewish (0.2\%; Hackett et al. 2012). In the future, the global religious trends projected by the Pew Research Center predict that by 2050 religiosity will increase in all the major religious groups, except the Buddhist. Even atheists, agnostics and people that are not affiliate to any religion, will represent a declining share of the world's total population (Pew Research Center Religion and Public Life 2015).

Iannaccone (2006) defines religion as 'any shared set of beliefs activities and institutions premised upon faith in supernatural forces'. Scholars pertaining to different social sciences disciplines have studied religions, as a form of magical supernaturalism, and its relationship with science and economy. Some key influential authors reach similar results about the inevitable decline of religion due to the rationalization process inherent to capitalism (Bever and Styers 2017). Religion, as a complex social phenomenon, has an effect on the economy (Parboteeah et al. 2015). Karl Marx attempted to examine religion from a scientific perspective, stating that religion is an expression of material realities and economic injustice. In his opinion, religion provides reasons to keep capitalism working. When capitalism stagnates and rising living standards cannot be guaranteed, religion fades (Marx and Stone 2014). In 1905, Max Weber argued that hard work and frugality eased the growth of capitalism in Western Europe (Weber 1930). For Weber, the "disenchantment" of the world becomes 
apparent when religion is substituted by science in order to explain questions of values and morals (Davis 2017; Weber 1946). Durkheim (1925) proposed that in modern societies, science would take the role of religion and socialism would replace Catholicism. For him, the division of labor, an economic arrangement, would bring along genuine morality (Durkheim 1926). Since then, many investigations have been carried out trying to establish relations between religion and various and more precise aspects related to the economy. Iyer (2016) made an extensive literature review of them concluding that a new field of inquiry, called the new economics of religion, has arisen (McCleary 2011).

One of the promising areas where research has recently and rapidly increased is the study of the relationship between religion and innovation (Bénabou et al. 2013, 2015; Barro and McCleary 2003; Guiso et al. 2003; Cavalcanti et al. 2007; Becker and Woessmann 2009; Levy and Razin 2012; Stark et al. 1996; Yerxa 2016; McCleary and Barro 2019; Carpantier and Litina 2019; Peifer et al. 2019; Liu et al. 2018; Basedau et al. 2018; Sherkat 2017; Huang et al. 2016). Religion has an impact on innovation, as has been demonstrated by different investigations. In Bénabou et al. (2013), they uncovered a robust negative association between religiosity and innovation, holding across countries ${ }^{1}$ as well as US states. In a more recent study, Bénabou et al. (2015), using all five waves of the World Values Survey, examined the relationships between eleven indicators of openness to innovation and five measures of religiosity. They found that greater religiosity was almost uniformly and very significantly associated to less favorable views of innovation. Our paper contributes to the literature of economics of religion in the context of innovation.

The future of firms and countries is closely related to their capacity to innovate (OECD 2015). A position as an innovative player will be necessary to gain a sustainable competitive advantage in the global markets (Damm et al. 2019). From a European perspective, it seems relevant to study the extent to which religious beliefs, and their associated practices, could favor or curb creativity and innovation (European Commission 2015). Furthermore, it also seems important to estimate what will be the projected evolution of that relationship in the coming years so that the economic, political and social actors can consider it (Center for Religion Studies. Fondazione Bruno Kessler 2018). Additionally, the Christian Churches having had a dominant position in social life in Europe (Pals 2006), it is interesting to study how new religious movements and new forms of religions behave towards economic innovation issues in the countries that belong to the European Union (Cooper and Patrick-Morsman 2019). Results shed some light on the debate about the European religion exceptionalism that stresses the peculiarity of the European experience with respect to the relationship between modernization and secularization (Davie 2002).

After an in-depth literature review, we propose two hypotheses about the relation between religiosity and innovation. The first one states that there is a negative relationship between religiosity and attitudes towards innovation. The second one says that innovativeness orientation depends on religion denomination.

We use the Eurobarometer 63.4 European Union Enlargement (European Communities 2005), the European Constitution, Economic Challenges, Innovative Products and Services for testing our hypotheses. This round of Eurobarometer surveys queried respondents on standard Eurobarometer measures as well as questions about innovative products or services. The survey also contains several demographic characteristics of respondents.

In order to explore if the relationships stated in the hypotheses hold, we perform a logistic regression and interpret the results using their odds-ratios and marginal effects.

Finally, the profiles obtained are connected to the innovations' product life-cycle. Managers obtain a clear link between religion and innovations that allows them to better manage different innovations' evolutive stages attending to the religion of their customers. The European policy-makers,

1 Countries under study were those with information about number of patents filed by both residents and foreigners in the World Intellectual Policy Organization (WIPO) database for the years 1980, 1990, 1995 and 2000. For more details see Bénabou et al. (2013). 
still dominated by a traditional innovation approach, gain a demand-side perspective to improve citizen's innovativeness awareness and acceptance.

\section{Literature Review and Hypotheses Development}

\subsection{Evolution of Religiosity}

Secularization has been a recurring theme in the sociology of religion (Pals 2006). Following the secularization hypothesis (Norris and Inglehart 2011), we would expect a decreased religiosity due to the effects of economic development and the associated increasing education and urbanization. Previous investigations (Finke 2013; Iannaccone 1998) conclude that almost all advanced industrial societies are evolving towards more secular orientations, yet the world as a whole has more people with traditional beliefs than ever before and it seems set to remain so, or even increase, in the near future (Pew Research Center Religion and Public Life 2015). Nevertheless, there is no general consensus among scholars about the secularization theories (Zielińska 2013). The defenders of the secularization thesis emphasize the loss of the sway and influence of religions (mainly Christian in Europe) in social life (Bruce 1992, 2002, 2011, 2013). Critics, through the rational choice theory of religion, see the secularization as the effect of a change at the individual level (individual beliefs, values, affiliations, practices and religious rites), not at a societal one, as the defenders do (Stark 1992, 1999). They also argue that modernization transforms religion, giving rise to the appearance of new religions or transforming the existing ones (Crippen 1988). In their view, the situation of religiosity outside Europe does not confirm the predictions of the secularization theories. It is the case the revitalization of Islam, in its most puritan versions, in various countries of the Middle East region (Gellner 1992), and the success of the conservative Protestantism in Latin America, Sub-Saharan Africa and Southern Asia (Zielińska 2013).

Some explanations for the failure of the envisioned secularization process can be found in the writings of the 'founding fathers' of sociology (Pals 2006). The differences between the two opposite positions found on the secularization theory stem from the difficulty in defining what religion is. Marx (1843) proposed that religion, better than anything else, addresses the emotional needs of an alienated and unhappy humanity. Some influential economists argue that the economic impact of the today's innovations may pale in comparison with those of the past, and are having high negative effects on social (v. gr., impact of robotization on working conditions) and environmental issues (v. gr., climate change; The Economist 2013, 2019). This 'innovation pessimism' could be the reason why religion is gaining momentum in society again. Weber (1978) maintained that the definition of religion could only be elaborated at the end of a study, rather than at its inception. It means, in our case, not only studying the Christian Churches but any other new religion movement or form. Furthermore, (Durkheim 1925) remarked the limitations stemming from the cultural embeddedness of defining concepts, which have to be universal. In this sense, the secularization hypothesis could not be generalized from the European experience of Christian Churches to the rest of the world and religions because it is affected by an ethnocentric approach.

Despite much of the research about religion and innovation is being based on U.S. data, investigations on Europe show that secularization is greater within its territory due to supply and demand effects (McBride 2010). A parallel question is the role of religion pluralism, religious participation and its consequences in the societies and economic outcomes. We found results pointing in two opposite directions: some authors say that pluralism reduces religious activity and so it fosters the secularization process (Montgomery 2003), while others maintain the opposite: pluralism intensifies religiosity (Finke and Stark 1988).

We need to understand which factors are associated with the increasing future role of religions in the world, and particularly in Europe. The rational choice approach says that individuals are rational consumers that behave and make decisions following a cost-benefit analysis even in religious affairs (Iannaccone 1992). A model proposed by Azzi and Ehrenberg (1975) identifies three motives for church 
attendance: "salvation motive" (expected afterlife consumption), a "consumption motive" (satisfaction with current church attendance) and a "social-pressure motive" (where church attendance directly increases the chance of success in business). The latter is the more interesting for our purposes. In order to reach a deeper insight of the aforementioned, the Club Goods Model explains how people embrace a religion based on social-pressures (Iannaccone 1992). This model considers religion as a club where people who belong to it obtain certain benefits. A distinction between churches and sects is also made, where the former are more consistent with the established criteria and the latter the more deviant. Sects usually attract people with fewer opportunities in the economic development process. The globalization that we have been experiencing in the previous three decades has brought continuous increases in the economic growth rate, but also has widened economic inequality within the most developed countries (Milanovic 2012). This reason could explain the increasing religiosity and extremism in Europe, and in other advanced industrial areas.

Innovation acceptance (or innovativeness, the term that we most like) could be seriously affected in the near future by these exclusion reasons. Technologies that have been gestating for years (for instance, robotics, next-generation genomics, artificial intelligence, automation of knowledge work, Internet of the Things and some others) will be part of our everyday life in the short term (Manyika et al. 2013). We could even expect diverse scenarios of religion strictness depending on the degree of the inequality and exclusion that the innovation process will imply (Levy and Razin 2012).

In that political, social and economic context, it seems necessary to analyze the coevolution of religious beliefs and scientific-innovative development. Churches are subject to the forces of competition and collaboration like any other economic or social subject. Smith (1776) maintained that clergy follow self-interest, and that market forces constrain them as they do with secular firms.

\subsection{Reasons Why Religiosity in General May Influence Attitudes towards Innovation}

According to the Oxford Dictionary (Simpson et al. 1989), innovation means to: "Make changes in something established, especially by introducing new methods, ideas, or products". In view of this definition, it is not strange that the relationship between religion (old/traditional) and innovation (new) has been tense. More concretely, we focus on innovation's economic and social perspective. Thus, innovation could be well defined as transforming an idea into a solution that incorporates added value from the client's point of view. Innovation feeds economic growth from progress in the basic sciences to the diffusion of new technologies, economic practices and even social change (Bénabou et al. 2015).

Governments, business and society tend to be in favor of more innovation, based on the belief that it leaves behind far more winners than losers (Mulgan 2016). This belief is known as the pro-innovation bias (Rogers 1995). To define innovation only considering its positive outcomes is not coherent with the negative results obtained from some innovations in the past (Rotman 2013). Very few articles consider the negative influence of innovation (Niilola 2017). Rogers (1995) said that 'negative effects of innovation are often unanticipated and most of the undesirable consequences emerge when the innovation is widely diffused and never comes to the attention of the innovator'. Nevertheless, it is necessary to differentiate between unanticipated and unintended (de Zwart 2015; Lazarus 2019). Following Niilola (2018), we could see that pioneers adopting an innovation might only use the product as it was originally intended, but the following adopters find other uses resulting in unanticipated and undesirable events. In order to fix this issue, the European Commission has promised to back responsible innovation, defined as 'an approach that anticipates and assesses potential implications and societal expectations with regard to research and innovation, with the aim to foster the design of inclusive and sustainable research and innovation' (European Commission 2009). This concept of innovation overcomes the pro-innovation bias found in previous definitions, but the process for how the ex-ante evaluation has to be done it is not an easy task because no one can predict how innovations will evolve or even predict who will benefit or will suffer from their outcomes (Mulgan 2016). The existence of the good vs. bad innovation dilemma will affect people behavior towards innovation. We can expect 
that the more an individual attends to their beliefs the more are likely to associate innovation outcomes with negative results because more religious people are more risk averse (Noussair et al. 2013).

Guiso et al. (2003) found that religiosity affects economic attitudes. They analyzed the relation between religion and six groups of variables: people's attitudes toward cooperation, women, government, legal rules, the market economy and its fairness and thriftiness. On average, religion is associated positively with attitudes that are conducive to free markets and better institutions. Different religions and the intensity of religious beliefs influence the results: Christians are more positively associated with attitudes that are conducive to economic growth, while Islam is negatively associated. The only case in which Protestants seem more pro-market than Catholics is on incentives; trust and intolerance toward immigrants is associated with worshippers.

The relationship between religion and innovation is mediated by science. Religion and science are generally considered incompatible. Commonly, religion is viewed as unscientific and the conflict arises because religious dogmas and practices are less believable when scientific discoveries occur (Brooks 1994). Innovation involves much more than the research and development activities (for instance: conception, initial knowledge generation, product engineering, manufacturing and marketing). Most people are aware of scientific progress when innovation transforms knowledge into commercial products.

Religiosity has an impact on innovation as has been demonstrated by different investigations (Assouad and Parboteeah 2018; Parboteeah et al. 2015; Audretsch et al. 2007). In Bénabou et al. (2013), they uncovered a robust negative association between religiosity and innovation, holding across countries as well as US states. In another study, Bénabou et al. (2015), using all five waves of the World Values Survey, examined the relationships between eleven indicators of openness to innovation and five measures of religiosity. They found that greater religiosity was almost uniformly and very significantly associated to less favorable views of innovation.

Therefore, we proposed the following hypothesis:

H1: There is a negative relationship between religiosity and attitudes towards innovation.

\subsection{Religions and Attitudes toward Innovation}

From a theoretical perspective, we are interested in the influence potential of religions on innovation. Eisenstadt (1968) says that this potential refers to "capacity to legitimize, in religious or ideological terms, the development of new motivations, activities, and institutions which were not encompassed by their original impulses and views.". His approach allows assessing the potential impact that any religion could have on economic activity. For instance, Hinduism does not have a clear position on economic activity, but the multiplicity of gods and sects and the ritualistic behavior promoted in this religion might not facilitate any innovation effort in order to obtain economic outcomes. However, the right interpretation of how this religion negatively affects any economic activity must be found, not in the sacred texts that support their faith, but in the inflexible institutions and behaviors that their followers design to discourage new growth values that put in danger the pre-existing status quo (Guiso et al. 2003).

More recent studies address how different faiths approach this question. Michalopoulos et al. (2016) provide a conceptual framework to think about how Islam is related to inequality and preindustrial comparative development. They show that for Islam, inequality has to be restrained in order to survive. They try to implement an income redistribution through religious strictures on capital accumulation and increased good provision from religious institutions. Conflict and cooperation have been studied in the Jewish communes as an example of organizations that stress cooperation and minimize conflict among members (Abramitzky 2008). It is also well documented how Christianity has influenced the innovation-religion relationship through, for instance, the ancient Inquisition institution (Findlen and Marcus 2012), enforced laws against usury (Rubin 2011) or a wide variety of religiously provided public services (education, elderly care, health care and some other social services; Hungerman 2005). Finally, as we already know, Guiso et al. (2003) found that Christians and Islamists have different attitudes towards economic growth, therefore the same could be expected in their acceptance about innovation. 
Consequently, we proposed the following hypothesis:

H2: Innovativeness orientation depends on religion denomination.

\section{Data and Methodology}

\subsection{The Sample}

The data comes from the EUROBAROMETER 63.4 that was carried out between the 9th of May and the 14th of June 2005, at the request of the EUROPEAN COMMISSION. The dataset was accessed through GESIS (Leibniz-Institute für Sozialwissenschaften). The EUROBAROMETER is part of wave 63.4 and covers the population of the respective nationalities of the European Union member states, resident in each of the member states and aged 15 years and over. The EUROBAROMETER 63.4 has also been conducted in the four candidate countries (Bulgaria, Croatia, Romania and Turkey) and in the northern part of Cyprus. In these countries, the survey covers the national population of citizens of the respective nationalities and the population of citizens of all the European Union member states that are resident in those countries and have a sufficient command of one of the respective national language(s) to answer the questionnaire. The basic sample design applied in all states is a multi-stage, random (probability) one.

The sample represents the whole territory of the countries surveyed according to the distribution of the resident population of the respective nationalities in terms of metropolitan, urban and rural areas. In each of the selected sampling points, a starting address was drawn, at random. Further addresses (every Nth address) were selected by standard "random route" procedures, from the initial address. In each household, the respondent was drawn, at random (following the "closest birthday rule"). All interviews were conducted face-to-face in people's homes and in the appropriate national language.

The following table (Table 1 ) shows the sample size in each country and the total population aged 15 or more years.

Table 1. Sample size by country, total population $15+$.

\begin{tabular}{|c|c|c|}
\hline Country & Number of Interviews & Population 15+ \\
\hline Austria & 1.000 & 6679.444 \\
\hline Belgium & 1.000 & 8508.982 \\
\hline Bulgaria & 1.018 & 6695.512 \\
\hline Croatia & 1.000 & 3682.826 \\
\hline Czech Republic & 1.083 & 8757.710 \\
\hline Denmark & 1.051 & 4380.063 \\
\hline Estonia & 1.001 & 8867.094 \\
\hline Finland & 1.024 & 4279.286 \\
\hline France & 1.012 & $44,010.619$ \\
\hline Germany & 1.520 & $64,174.295$ \\
\hline Greece & 1.000 & 8674.230 \\
\hline Hungary & 1.014 & 8503.379 \\
\hline Ireland & 1.006 & 3089.775 \\
\hline Italy & 1.004 & $49,208.000$ \\
\hline Latvia & 1.015 & 1394.351 \\
\hline Lithuani & 1.003 & 2803.661 \\
\hline Luxemburg & 504 & 367.199 \\
\hline Malta & 500 & 322.917 \\
\hline The Netherlands & 1.006 & $13,242.328$ \\
\hline North part of Cyprus & 500 & 157.101 \\
\hline Poland & 1.000 & $31,610.437$ \\
\hline Portugal & 1.005 & 8080.915 \\
\hline Republic of Cyprus & 505 & 552.213 \\
\hline Romania & 1.004 & $18,145.036$ \\
\hline Slovakia & 1.108 & 4316.438 \\
\hline Slovenia & 1.045 & 1663.869 \\
\hline Spain & 1.024 & $35,882.820$ \\
\hline Sweden & 1.024 & 7376.680 \\
\hline Turkey & 1.005 & $47,583.830$ \\
\hline United Kingdom & 1.347 & $47,685.578$ \\
\hline Total & 29.328 & $442,620.588$ \\
\hline
\end{tabular}


The sample adequately represents the share of the different religious groups in Europe. In the next table (Table 2), we could compare the percentages in the sample used and the estimations made by Pew Research Center Religion and Public Life (2015) for Europe in the year 2010. All major religions were well represented in the sample, and only minority religions (Buddhist, Jewish and Hindu) were slightly under-represented.

Table 2. Sample representativeness of European religions.

\begin{tabular}{|c|c|c|c|c|}
\hline \multicolumn{2}{|c|}{ Religion } & Number of Interviews & Percent & Pew Research Percent Estimation \\
\hline \multirow{4}{*}{ Christians } & Catholic & 13.191 & 45 & \multirow{4}{*}{74.5} \\
\hline & Orthodox & 3.719 & 12.7 & \\
\hline & Protestant & 4.100 & 14 & \\
\hline & Other Christian & 1.063 & 3.6 & \\
\hline \multicolumn{2}{|l|}{ Jewish } & 25 & 0.1 & 0.2 \\
\hline \multicolumn{2}{|l|}{ Muslim } & 1.747 & 6 & 5.9 \\
\hline \multicolumn{2}{|l|}{ Sikh } & 8 & 0 & - \\
\hline \multicolumn{2}{|l|}{ Buddhist } & 41 & 0.1 & 0.2 \\
\hline \multicolumn{2}{|l|}{ Hindu } & 16 & 0.1 & 0.2 \\
\hline \multicolumn{2}{|l|}{ Atheist } & 1.316 & 4.5 & \multirow{2}{*}{18.8} \\
\hline \multicolumn{2}{|c|}{ Non believer /agnostic } & 3.253 & 11.1 & \\
\hline \multicolumn{2}{|c|}{ Other (SPONT.) } & 415 & 1.4 & - \\
\hline \multicolumn{2}{|c|}{ Do not know (DK) } & 434 & 1.5 & - \\
\hline \multicolumn{2}{|l|}{ Total } & 29.328 & 100 & 100 \\
\hline
\end{tabular}

Source: Own elaboration based on European Communities (2005) and Pew Research Center Religion and Public Life (2015).

- Thirteen questions about respondents' beliefs, attitudes and behaviors towards innovation and innovative products-attraction to innovations, purchasing intention, innovation meaning, innovative buying preference, price, innovation as gadgets, innovations as fashion and innovations simplifying everyday life (dependent variables);

- Two questions regarding religiosity - the first, to what religion faith the interviewee belongs; and the second, the frequency which they attend religious acts (independent variables);

- Finally, five sociodemographic aspects that arose in the literature review as having a potential effect on the dependent variables under study - marital status, education, gender, age and type of community where the interviewee lives (control variables).

For innovative products' variables that have more than two possible categories of responses-attraction, purchase and price-we have recoded them in two groups (high and low), creating a dummy variable equal to one, if the response is in the high group, and zero otherwise. The final sample for analysis excludes all the missing values and religions with a low rate of respondents-Jewish, Sikh, Buddhist, Hindu and other². Sample descriptives are shown in Table 3.

2 In order to decide which variables to include and which categories to delete, we ran an exploratory analysis using the MOSAIC technique included in the STRUCTPLOT FRAMEWORK developed in R software. Minor Religions in Europe-Jews, Buddhists, Hindus and Sikhs - and people that spontaneously manifest belonging to a different religion, do not show any remarkable pattern in their standardized residuals analysis. There are few observations in the sample to obtain significant statistical results in all the analysis that we run. Hence, we decided not to consider them for analysis. 
Table 3. Sample descriptives.

\begin{tabular}{|c|c|c|c|c|c|c|c|c|c|}
\hline Variable & Categories (Original Scale) & $\begin{array}{l}\text { n (Original } \\
\text { Sample) }\end{array}$ & $\begin{array}{c}\text { Orginal } \\
\text { Sample Share }\end{array}$ & $\begin{array}{l}\text { Categories } \\
\text { (Analysis) }\end{array}$ & n (Analysis) & $\begin{array}{l}\text { Final Analysis } \\
\text { Sample Share }\end{array}$ & Operationalization & Value & Question \\
\hline \multirow{4}{*}{$\begin{array}{l}\text { Attitude } \\
\text { 1. Attraction }\end{array}$} & purchasing behavior towards innova & a (dependent $v a$ & ables) & & & & & & \multirow{4}{*}{$\begin{array}{l}\text { In general, to what extent are you } \\
\text { attracted towards innovative } \\
\text { products or services, in other words } \\
\text { new or improved products or } \\
\text { services? }\end{array}$} \\
\hline & Very Attracted & 3.825 & 13.0 & Attracted & 16.554 & 59.0 & \multirow{3}{*}{$\begin{array}{l}\text { Reference } \\
\text { category }\end{array}$} & 1 & \\
\hline & Fairly attracted & 12.729 & 43.4 & Not ATTRACTED & 11.485 & 41.0 & & 0 & \\
\hline & $\begin{array}{l}\text { Not at all attracted } \\
\text { DK (missing) }\end{array}$ & $\begin{array}{l}3.354 \\
1.289\end{array}$ & $\begin{array}{c}11.4 \\
4.4\end{array}$ & & & & & & \\
\hline \multirow[t]{2}{*}{ 2. Purchase } & More inclined to purchase & 7.745 & 26.4 & More inclined to & 7.745 & 28.4 & \multirow[b]{2}{*}{$\begin{array}{l}\text { Reference } \\
\text { category }\end{array}$} & 1 & \multirow{2}{*}{$\begin{array}{l}\text { Compared to your friends and } \\
\text { family, would you say that you tend } \\
\text { to be } \ldots \text { ? }\end{array}$} \\
\hline & Less inclined to purchase & 11.919 & 40.6 & $\begin{array}{l}\text { As or less inclined to } \\
\text { purchase }\end{array}$ & 19.544 & 71.6 & & 0 & \\
\hline \multirow[t]{3}{*}{ 3. Meaning } & $\begin{array}{l}\text { The creation of new products } \\
\text { and services }\end{array}$ & 14.893 & 50.8 & $\begin{array}{l}\text { The creation of new } \\
\text { products and services }\end{array}$ & 14.893 & 56.7 & & 1 & \multirow{3}{*}{$\begin{array}{l}\text { What does "innovation" mean for } \\
\text { you? The creation of new products } \\
\text { or services or the improvement of } \\
\text { existing products or services? }\end{array}$} \\
\hline & $\begin{array}{l}\text { The improvement of existing } \\
\text { products and services }\end{array}$ & 11.395 & 38.9 & $\begin{array}{l}\text { The improvement of } \\
\text { existing products and } \\
\text { services }\end{array}$ & 11.395 & 43.3 & $\begin{array}{l}\text { Reference } \\
\text { category }\end{array}$ & 0 & \\
\hline & DK (missing) & 3.040 & 10.4 & & & & & & \\
\hline \multirow[t]{3}{*}{ 4. Buy } & Do not try the innovative one. & 12.736 & 43.4 & $\begin{array}{l}\text { Do not try the } \\
\text { innovative one. }\end{array}$ & 12.736 & 47,4 & \multirow[t]{3}{*}{$\begin{array}{l}\text { Reference } \\
\text { category }\end{array}$} & 0 & \multirow{3}{*}{$\begin{array}{l}\text { In general, when an innovative } \\
\text { product or service is put on the } \\
\text { market and can replace a product or } \\
\text { service that you already trust...? }\end{array}$} \\
\hline & Try the innovative product & 14.128 & 48.2 & $\begin{array}{l}\text { Try the innovative } \\
\text { product }\end{array}$ & 14.128 & 52.6 & & 1 & \\
\hline & DK (missing) & 2.464 & 8.4 & & & & & & \\
\hline \multirow{3}{*}{ 5. Price } & Only if little more expensive. & 8.940 & 30.5 & $\begin{array}{l}\text { Never or only if cost } \\
\text { the same }\end{array}$ & 16.204 & 60.0 & \multirow[t]{3}{*}{$\begin{array}{l}\text { Reference } \\
\text { category }\end{array}$} & 0 & \multirow{3}{*}{$\begin{array}{c}\text { You would be willing to replace a } \\
\text { product or a service that you already } \\
\text { use by an innovative one ... }\end{array}$} \\
\hline & Only if cost the same. & 13.232 & 45.1 & & & & & & \\
\hline & $\begin{array}{l}\text { Never purchase } \\
\text { DK (missing) }\end{array}$ & $\begin{array}{l}2.972 \\
2.303\end{array}$ & $\begin{array}{l}10.1 \\
7.9\end{array}$ & & & & & & \\
\hline \multirow[t]{2}{*}{ 6. Gadgets } & Mentioned & 23.573 & 80.4 & Mentioned & 23.573 & 80.4 & \multirow[b]{2}{*}{$\begin{array}{l}\text { Reference } \\
\text { category }\end{array}$} & 1 & \multirow{2}{*}{$\begin{array}{l}\text { Innovative products or services are } \\
\text { most of the time gadgets }\end{array}$} \\
\hline & Not mentioned & 5.755 & 19.6 & Not mentioned & 5.755 & 19.6 & & 0 & \\
\hline \multirow[t]{2}{*}{ 7. Fashion } & Mentioned & 20.569 & 70.1 & Mentioned & 20.569 & 70.1 & \multirow[b]{2}{*}{$\begin{array}{l}\text { Reference } \\
\text { category }\end{array}$} & 1 & \multirow{2}{*}{$\begin{array}{l}\text { Innovative products or services are a } \\
\text { matter of fashion }\end{array}$} \\
\hline & Not mentioned & 8.759 & 29.9 & Not mentioned & 8.759 & 29.9 & & 0 & \\
\hline \multirow{2}{*}{ 8. Simplify } & Mentioned & 17.288 & 58.9 & Mentioned & 17.288 & 58.9 & \multirow{2}{*}{$\begin{array}{l}\text { Reference } \\
\text { category }\end{array}$} & 1 & \multirow{2}{*}{$\begin{array}{l}\text { Innovative products or services } \\
\text { often simplify everyday life }\end{array}$} \\
\hline & Not mentioned & 12.040 & 41.1 & Not mentioned & 12.040 & 41.1 & & 0 & \\
\hline \multirow[t]{2}{*}{ 9. Image } & Mentioned & 20.937 & 71.4 & Mentioned & 20.937 & 71.4 & \multirow[b]{2}{*}{$\begin{array}{l}\text { Reference } \\
\text { category }\end{array}$} & 1 & \multirow{2}{*}{$\begin{array}{l}\text { A company that sells an innovative } \\
\text { product or service improves the } \\
\text { image of all its products or services }\end{array}$} \\
\hline & Not mentioned & 8.391 & 28.6 & Not mentioned & 8.391 & 28.6 & & 0 & \\
\hline
\end{tabular}


Table 3. Cont

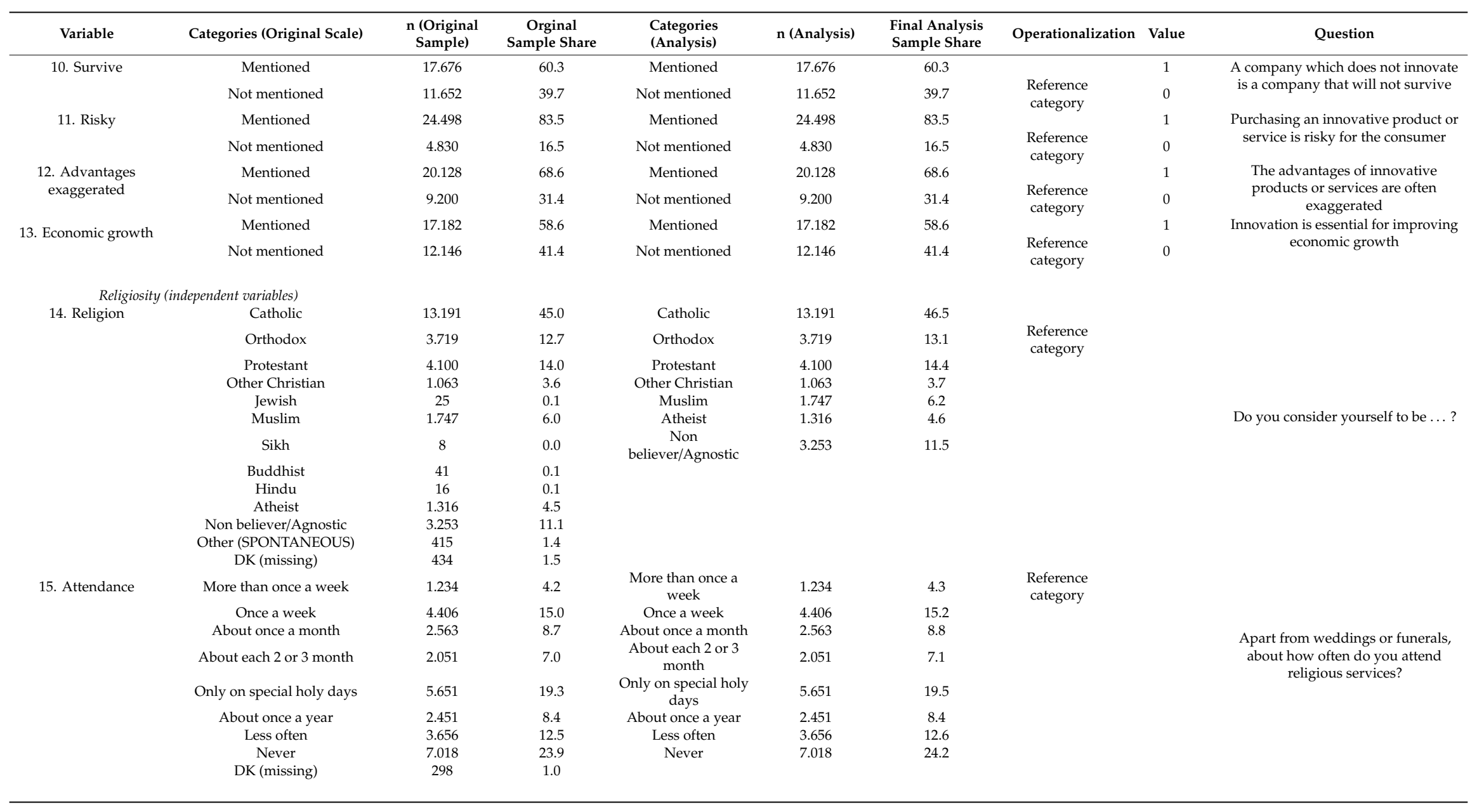


Table 3. Cont

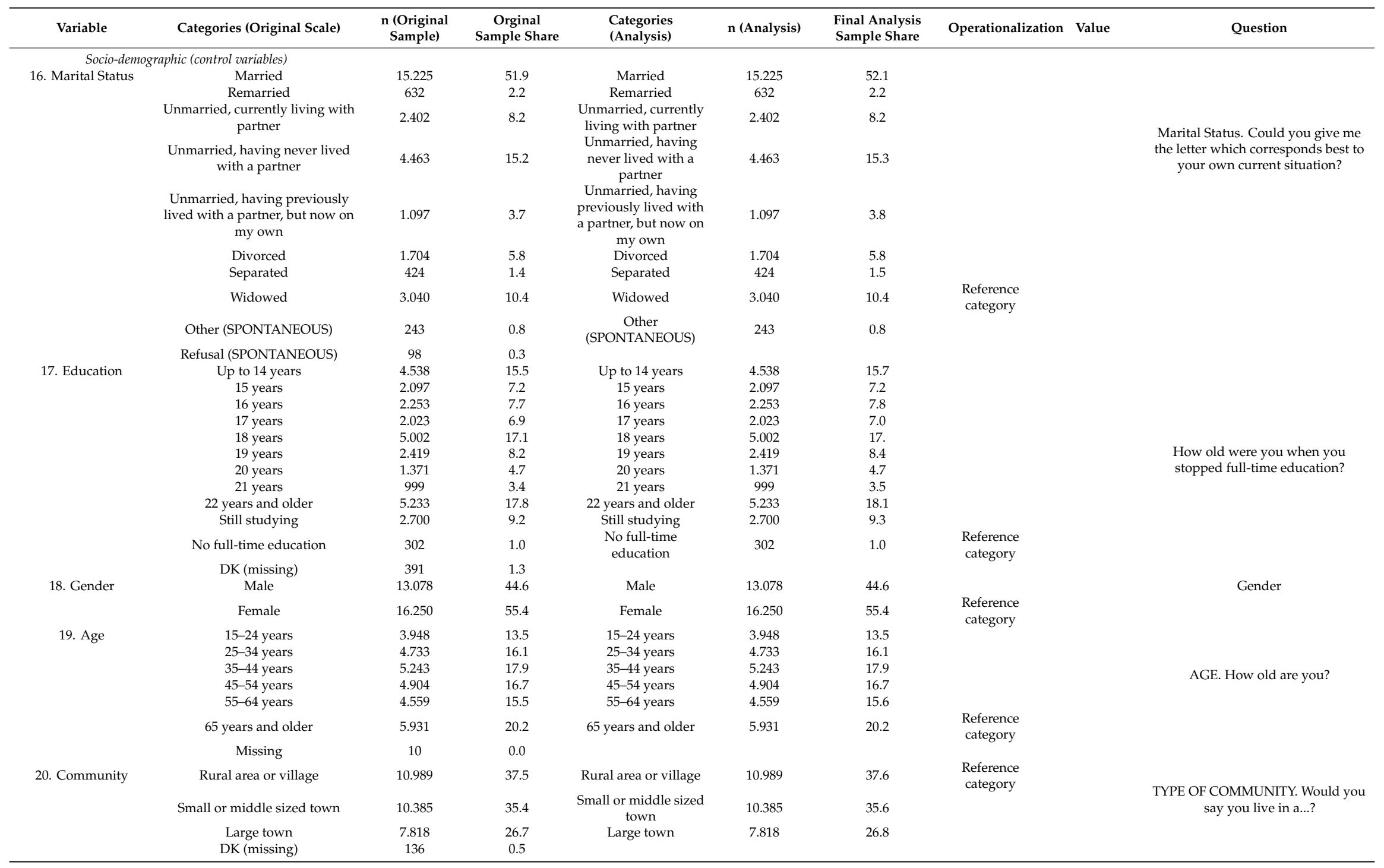




\subsection{Methodology}

Considering that all the variables are categorical in nature, and all dependent variables are binary (after converting three of them from multinomial to binary as we saw in Table 3), in order to confirm the multivariate relationships stated in the hypotheses, we select a logistic regression approach.

With the intention of improving the interpretability of the regression coefficients, we use marginal effects. Marginal effect is a measure of the instantaneous effect that a change in a particular explanatory variable has on the predicted probability of the dependent variable, when the other covariates are kept fixed (SAS 2018). The dependent variable is modeled as follows:

$$
y=E(y / x)+\varepsilon
$$

where $E(y / x)$ is the conditional mean function, $x$ is the vector of explanatory variables and $\varepsilon$ is the error term. The conditional mean function is given by:

$$
E(y / x)=F\left(\beta^{\prime} x\right)
$$

where $\boldsymbol{F}$ denotes a cumulative distribution function and $\beta$ denotes the parameters. Therefore,

$$
\operatorname{Pr}(y=1)=F\left(\beta^{\prime} x\right)
$$

Marginal effects are obtained by computing the derivative of the conditional mean function with respect to given by:

$$
\frac{\partial E(y / x)}{\partial x}=\left[\frac{\partial F\left(\beta^{\prime} x\right)}{\partial \beta^{\prime} x}\right] \beta=f\left(\beta^{\prime} x\right) \beta,
$$

where $f($.$) is the density function that corresponds to the cumulative function F($.$) . In this study,$ where all the variables are categorical (mostly binary), we would report the difference between the estimated probability if the variable is equal to 1 and the estimated probability if the variable is equal to 0 . The marginal effects are nonlinear functions of the parameter estimates and levels of the explanatory variables. Hence, they generally cannot be inferred directly from parameter estimates. In this case, we used the R library called "margins".

As we have already seen, marginal effects can be defined as the change in the dependent variable as a function of the variation in other (one or more) independent variables of interest, while all other variables in the model remain constant. There are three different marginal effects approaches: marginal effects at the means (MEMs), average marginal effects (AMEs) and marginal effects at representative values (MERs). Following (Bartus 2005) recommendations, we used MERs because it allows the choice of a range of values for one or more independent variables, focusing on how marginal effects behave in that range.

For interpretation purposes, marginal effects are popular because they make available the amount of change in the dependent variable $(\mathrm{Y})$ that will be given by a 1-unit change in an independent variable $(X)$. For binary dependent and independent variables, the marginal effect shows how the probability of the dependent variable $\mathrm{P}(\mathrm{Y}=1)$ changes when the independent binary variable changes from 0 to 1 , after controlling the other variables in the model. When you have independent categorical variables, like in our case religion and religiosity, the marginal effects are interpreted as the difference in predicted probabilities for cases in one category relative to the reference category. In this paper, dummy variables were created for each of the categories of religion and religiosity and then marginal effects were calculated.

Before running the logistic regressions, we checked whether it would be possible to reduce the thirteen dependent variables to a lesser quantity. In doing so, we ran a factor analysis. Results showed that it was not adequate to reduce the dependent variables' dimensionality through a factor analysis because: 
- The tests in correlations were not significant in approximately $50 \%$ of the cases.

- There were three factors with eigenvalues greater than 1 that explained only $51 \%$ of the total variance, when the acceptable minimum is $60 \%$ (Hair et al. 2014).

- Finally, and most importantly, factors were not interpretable, even when the solution was rotated.

Consequently, we regressed thirteen variables related to innovation on two measures related to religion together with five sociodemographic controls using logistic regression.

\section{Results}

\subsection{Religion and Innovation Outcomes}

Results from the logistic regression applied on the three innovation outcomes variables considered can be seen in Table 4.

The Orthodox has the worst profile towards innovation outcomes: No other religion has odds smaller than 1 at significant levels, being this religion is the reference category.

Considering the company image odds, the top position was occupied by the Atheist (odds $=1.667$ ), followed by a second group composed of agnostic (odds $=1.312$ ), other Christian (odds =1.298), Protestant (odds $=1.236$ ) and other (SPONT.; odds $=1.303$ ). All the rest of religious affiliations had no significant result.

Table 4. Logistic regression results and innovation outcomes.

\begin{tabular}{|c|c|c|c|c|c|c|c|}
\hline & & \multicolumn{6}{|c|}{ Dependent Variable: } \\
\hline & & \multicolumn{2}{|c|}{ Company Image } & \multicolumn{2}{|c|}{ Company Survival } & \multicolumn{2}{|c|}{ Economic Growth } \\
\hline & & Log-Odds & Odds & Log-Odds & Odds & Log-Dds & Odds \\
\hline \multirow{4}{*}{$\begin{array}{l}\text { Religion } \\
\text { (Ref. cat. } \\
\text { Orthodox) }\end{array}$} & Catholic & $\begin{array}{c}0.066 \\
-0.044\end{array}$ & 1.068 & $\begin{array}{c}0.288^{* * *} \\
-0.042\end{array}$ & 1.334 & $\begin{array}{c}0.182^{* * *} \\
-0.041\end{array}$ & 1.200 \\
\hline & Non believer/Agnostic & $\begin{array}{c}0.272 * * * \\
-0.061\end{array}$ & 1.312 & $\begin{array}{c}0.538^{* * *} \\
-0.057\end{array}$ & 1.713 & $\begin{array}{c}0.539 * * * \\
-0.057\end{array}$ & 1.714 \\
\hline & Other (SPONT. & $\begin{array}{l}0.264 \text { ** } \\
-0.1179\end{array}$ & 1.303 & $\begin{array}{c}0.520 * * * \\
-0.111\end{array}$ & 1.683 & $\begin{array}{c}0.558^{* * *} \\
-0.111\end{array}$ & 1.747 \\
\hline & Other Christian & $\begin{array}{c}0.261^{* * *} \\
-0.079\end{array}$ & 1.298 & $\begin{array}{c}0.657^{* * *} \\
-0.074\end{array}$ & 1.930 & $\begin{array}{c}0.662^{* * *} \\
-0.074\end{array}$ & 1.939 \\
\hline \multirow{5}{*}{$\begin{array}{c}\text { Religiosity } \\
\text { (Ref. cat. More } \\
\text { than once a week) }\end{array}$} & Once a month & $\begin{array}{l}-0.058 \\
-0.082\end{array}$ & 0.944 & $\begin{array}{l}0.137 \text { * } \\
-0.076\end{array}$ & 1.147 & $\begin{array}{l}0.132 \text { * } \\
-0.077\end{array}$ & 1.141 \\
\hline & Each 2 or 3 month & $\begin{array}{c}0.095 \\
-0.084\end{array}$ & 1.100 & $\begin{array}{l}0.1707^{*} \\
-0.0789\end{array}$ & 1.100 & $\begin{array}{c}0.239 * * * \\
-0.077\end{array}$ & 1.270 \\
\hline & On special holy days & $\begin{array}{l}-0.015 \\
-0.075\end{array}$ & 0.985 & $\begin{array}{c}0.143^{* *} \\
-0.07\end{array}$ & 1.153 & $\begin{array}{c}0.188^{* * *} \\
-0.07\end{array}$ & 1.207 \\
\hline & Once a year & $\begin{array}{l}-0.066 \\
-0.083\end{array}$ & 0.940 & $\begin{array}{c}0.297^{* * *} \\
-0.077\end{array}$ & 1.345 & $\begin{array}{c}0.228^{* * *} \\
-0.078\end{array}$ & 1.256 \\
\hline & Less often & $\begin{array}{l}-0.098 \\
-0.079\end{array}$ & 0.907 & $\begin{array}{c}0.0567 \\
-0.7315\end{array}$ & 1.067 & $\begin{array}{c}0.075 \\
-0.074\end{array}$ & 1.078 \\
\hline
\end{tabular}


Table 4. Cont.

\begin{tabular}{|c|c|c|c|c|c|c|c|}
\hline & & \multicolumn{6}{|c|}{ Dependent Variable: } \\
\hline & & \multicolumn{2}{|c|}{ Company Image } & \multicolumn{2}{|c|}{ Company Survival } & \multicolumn{2}{|c|}{ Economic Growth } \\
\hline & & Log-Odds & Odds & Log-Odds & Odds & Log-Dds & Odds \\
\hline \multirow[t]{8}{*}{$\begin{array}{c}\text { Marital Status } \\
\text { (Ref. cat. Widowed) }\end{array}$} & Divorced & $\begin{array}{l}0.204^{* * *} \\
-0.078\end{array}$ & 1.226 & $\begin{array}{l}0.322 * * * \\
-0.069\end{array}$ & 1.383 & $\begin{array}{l}0.159 * * \\
-0.07\end{array}$ & 1.173 \\
\hline & Married & $\begin{array}{l}0.287^{* * *} \\
-0.057\end{array}$ & 1.332 & $\begin{array}{l}0.283^{* * *} \\
-0.05\end{array}$ & 1.328 & $\begin{array}{l}0.236^{* * *} \\
-0.05\end{array}$ & 1.266 \\
\hline & Remarried & $\begin{array}{l}0.440 * * * \\
-0.102\end{array}$ & 1.552 & $\begin{array}{l}0.340 * * * \\
-0.096\end{array}$ & 1.405 & $\begin{array}{l}0.284^{* *} \\
-0.096\end{array}$ & 1.328 \\
\hline & Separated & $\begin{array}{l}0.233 * \\
-0.125\end{array}$ & 1.263 & $\begin{array}{c}0.178 \\
-0.115\end{array}$ & 1.195 & $\begin{array}{c}0.113 \\
-0.115\end{array}$ & 1.120 \\
\hline & $\begin{array}{l}\text { Unmarried. } \\
\text { living with partner }\end{array}$ & $\begin{array}{l}0.328^{* * *} \\
-0.076\end{array}$ & 1.388 & $\begin{array}{l}0.250 * * * \\
-0.069\end{array}$ & 1.284 & $\begin{array}{l}0.266^{* * *} \\
-0.069\end{array}$ & 1.304 \\
\hline & $\begin{array}{l}\text { Unmarried. } \\
\text { never lived with } \\
\text { partner }\end{array}$ & $\begin{array}{l}0.328^{* * *} \\
-0.076\end{array}$ & 1.282 & $\begin{array}{l}0.305^{* * *} \\
-0.0672\end{array}$ & 1.356 & $\begin{array}{l}0.196^{* * *} \\
-0.067\end{array}$ & 1.216 \\
\hline & $\begin{array}{l}\text { Unmarried. } \\
\text { previously lived with } \\
\text { partner }\end{array}$ & $\begin{array}{l}0.252 * * * \\
-0.074\end{array}$ & 1.300 & $\begin{array}{c}0.301 * * * \\
-0.082\end{array}$ & 1.352 & $\begin{array}{l}0.200 * * \\
-0.082\end{array}$ & 1.221 \\
\hline & $\begin{array}{c}\text { Other } \\
\text { (SPONTANEOUS }\end{array}$ & $\begin{array}{c}0.001 \\
-0.167\end{array}$ & 1.001 & $\begin{array}{c}0.094 \\
-0.152\end{array}$ & 1.098 & $\begin{array}{c}0.08 \\
-0.151\end{array}$ & 1.084 \\
\hline \multirow[t]{10}{*}{$\begin{array}{l}\text { Education } \\
\text { (Ref. cat. No full-time } \\
\text { education) }\end{array}$} & Up to 14 years & $\begin{array}{l}0.445^{* *} \\
-0.181\end{array}$ & 1.560 & $\begin{array}{l}0.315^{* *} \\
-0.148\end{array}$ & 1.370 & $\begin{array}{l}0.799 * * * \\
-0.175\end{array}$ & 2.223 \\
\hline & 15 years & $\begin{array}{l}0.567^{* * *} \\
-0.185\end{array}$ & 1.763 & $\begin{array}{l}0.394^{* * *} \\
-0.152\end{array}$ & 1.483 & $\begin{array}{l}0.895^{* * *} \\
-0.179\end{array}$ & 2.448 \\
\hline & 16 years & $\begin{array}{l}0.674^{* * *} \\
-0.185\end{array}$ & 1.962 & $\begin{array}{l}0.564^{* * *} \\
-0.151\end{array}$ & 1.758 & $\begin{array}{l}1.153 * * * \\
-0.178\end{array}$ & 3.167 \\
\hline & 17 years & $\begin{array}{l}0.723^{* * *} \\
-0.185\end{array}$ & 2.061 & $\begin{array}{l}0.699 * * * \\
-0.152\end{array}$ & 2.012 & $\begin{array}{l}1.262^{* * *} \\
-0.179\end{array}$ & 3.533 \\
\hline & 18 years & $\begin{array}{c}0.7441 * * * \\
-0.1811\end{array}$ & 2.103 & $\begin{array}{l}0.690 * * * \\
-0.148\end{array}$ & 1.993 & $\begin{array}{l}1.303^{* * *} \\
-0.175\end{array}$ & 3.680 \\
\hline & 19 years & $\begin{array}{c}0.878^{* * *} \\
-0.184\end{array}$ & 2.405 & $\begin{array}{c}0.878^{* * *} \\
-0.151\end{array}$ & 2.407 & $\begin{array}{l}1.538^{* * *} \\
-0.178\end{array}$ & 4.656 \\
\hline & 20 years & $\begin{array}{l}0.884^{* * *} \\
-0.188\end{array}$ & 2.422 & $\begin{array}{l}0.919 * * * \\
-0.155\end{array}$ & 2.507 & $\begin{array}{c}1.502 * * * \\
-0.181\end{array}$ & 4.490 \\
\hline & 21 years & $\begin{array}{c}0.879 * * * \\
-0.192\end{array}$ & 2.409 & $\begin{array}{c}1.061^{* * *} \\
-0.159\end{array}$ & 2.889 & $\begin{array}{l}1.617^{* * *} \\
-0.185\end{array}$ & 5.038 \\
\hline & 22 years and older & $\begin{array}{c}1.039^{* * *} \\
-0.181\end{array}$ & 2.828 & $\begin{array}{l}0.991^{* * *} \\
-0.148\end{array}$ & 2.694 & $\begin{array}{l}1.765^{* * *} \\
-0.175\end{array}$ & 5.844 \\
\hline & Still studying & $\begin{array}{l}0.974^{* * *} \\
-0.1896\end{array}$ & 2.684 & $\begin{array}{l}0.849 * * * \\
-0.158\end{array}$ & 2.337 & $\begin{array}{l}1.493^{* * *} \\
-0.183\end{array}$ & 4.452 \\
\hline \multirow{6}{*}{$\begin{array}{c}\text { Sex } \\
\text { (Ref. cat. Female) } \\
\text { Age } \\
\text { (Ref. cat. } 65 \text { years and } \\
\text { older) }\end{array}$} & Male & $\begin{array}{c}0.180^{* * *} \\
-0.028\end{array}$ & 1.198 & $\begin{array}{l}0.159 * * * \\
-0.026\end{array}$ & 1.172 & $\begin{array}{l}0.203 * * * \\
-0.026\end{array}$ & 1.225 \\
\hline & 15-24 years & $\begin{array}{l}0.326^{* * *} \\
-0.074\end{array}$ & 1.386 & $\begin{array}{c}0.022 \\
-0.069\end{array}$ & 1.022 & $\begin{array}{l}0.176 * \\
-0.069\end{array}$ & 1.193 \\
\hline & 25-34 years & $\begin{array}{l}0.360^{* * *} \\
-0.0542\end{array}$ & 1.433 & $\begin{array}{l}0.150 * * * \\
-0.05\end{array}$ & 1.162 & $\begin{array}{c}0.149^{* *} \\
-0.05\end{array}$ & 1.161 \\
\hline & $35-44$ years & $\begin{array}{l}0.328^{* * *} \\
-0.051\end{array}$ & 1.389 & $\begin{array}{l}0.210 * * * \\
-0.046\end{array}$ & 1.234 & $\begin{array}{l}0.198^{* * *} \\
-0.046\end{array}$ & 1.218 \\
\hline & $45-54$ years & $\begin{array}{l}0.311^{* * *} \\
-0.05\end{array}$ & 1.365 & $\begin{array}{l}0.206^{* * *} \\
-0.046\end{array}$ & 1.228 & $\begin{array}{l}0.247^{* * *} \\
-0.046\end{array}$ & 1.280 \\
\hline & 55-64 years & $\begin{array}{l}0.252^{* * *} \\
-0.05\end{array}$ & 1.286 & $\begin{array}{l}0.229 * * * \\
-0.045\end{array}$ & 1.257 & $\begin{array}{l}0.222 * * * \\
-0.045\end{array}$ & 1.249 \\
\hline \multirow[t]{6}{*}{$\begin{array}{c}\text { Community } \\
\text { (Ref. cat. Rural area) }\end{array}$} & Large town & $\begin{array}{l}0.099 * * * \\
-0.035\end{array}$ & 1.104 & $\begin{array}{l}0.068^{* *} \\
-0.0323\end{array}$ & 1.070 & $\begin{array}{l}0.0533 \\
-0.032\end{array}$ & 1.054 \\
\hline & $\begin{array}{c}\text { Small or middle sized } \\
\text { town }\end{array}$ & $\begin{array}{c}0.01 \\
-0.032\end{array}$ & 1.010 & $\begin{array}{c}0.02 \\
-0.029\end{array}$ & 1.021 & $\begin{array}{l}0.003 \\
-0.03\end{array}$ & 1.003 \\
\hline & Constant & $\begin{array}{l}-2.364^{* * *} \\
-0.197 \\
\end{array}$ & 0.094 & $\begin{array}{l}-2.105^{* * *} \\
-0.165\end{array}$ & 0.122 & $\begin{array}{l}-2.560^{* * *} \\
-0.19\end{array}$ & 0.077 \\
\hline & Observations & 28,052 & & 28,052 & & 28,052 & \\
\hline & Log Likelihood & $-16,443.05$ & & $-18,262.67$ & & $-18,206.29$ & \\
\hline & Akaike Inf. Crit. & $32,968.1$ & & $36,607.35$ & & $36,494.59$ & \\
\hline
\end{tabular}

Note: ${ }^{*} p<0.05 ;{ }^{* *} p<0.01 ;{ }^{* * *} p<0.001$.

In relation to company survival odds, Protestants had the highest probability perceiving of innovation as a key factor for businesses' future (odds $=2.055$ ). They were closely followed by other Christian (odds =1.930), Atheist (odds = 1.755), Agnostic (odds = 1.713) and other (SPONT.; odds $=1.683$ ). In the lower band we found Muslim (odds $=1.362$ ) and Catholic (odds $=1.334$ ). 
Regarding economic growth, two groups arose. The first one had higher odds considering that innovation impulses economic growth. It was formed of other Christian (odds $=1.939$ ), Atheist (odds $=1.910)$, Protestant (odds = 1.864), other (SPONT.; odds = 1.747) and Agnostic (odds = 1.714). The second group, with the lowest odds, was composed of Muslim (odds =1.271) and Catholic (odds $=1.200)$.

As result, innovation outcomes perceptions were affected according to religion affiliation. Atheists appeared in the optimistic leading group for the three variables considered and Orthodox, at the opposite end of the spectrum, had the lowest odds ratio (1). Among them, we found two different segments: one formed of those that scored high in the odds for the three variables (Agnostic, other Christian, Protestant and other (SPONT.)); and the other, with lower probabilities of considering innovation as a driver to obtain a company's improved image, to help companies to survive or to impulse economic growth (Catholics and Muslims).

\subsection{Innovation Outcomes Marginal Effects}

In order to better interpret these results we used marginal effects (MERs) on the logit models obtained for each of the innovation outcomes' dependent variables. It was computed using R's "margins" library (Version 0.3.20). We can see in Figure 1 that the pattern found through the odds from the logistic regression remained.

From Figure 1, we could deduce that, in relation to how innovation affects the company image, four groups of religions were conformed. The first one, formed by Atheists had marginal effects higher than 10\% (comparing to Orthodox) responding that innovation improved company image. Catholics and Muslims belonged to the second group, having a profile with marginal effects close to $0 \%$, meaning that they were very similar to Orthodox and manifested that company image was not significantly affected by innovation. Halfway between these two groups, with marginal effects about $5 \%$, were Agnostics, Protestants, other Christians and others. Religiosity (religion attendance) is also printed in Figure 1 and no association, or clear pattern, could be found.

As Figure 1 shows, religion affected the perception on innovation capacity to be associated with company survival. On the one hand, we found the group comprised of Protestants, other Christians, others, Atheists and Agnostics with marginal effects from 10\% to 17\% higher than Orthodox. On the other hand, with marginal effects around 5\%, appeared for Catholics and Muslims. As religiosity decreased there was a slight improvement of marginal effects, but it was not relevant enough to conclude any pattern. 


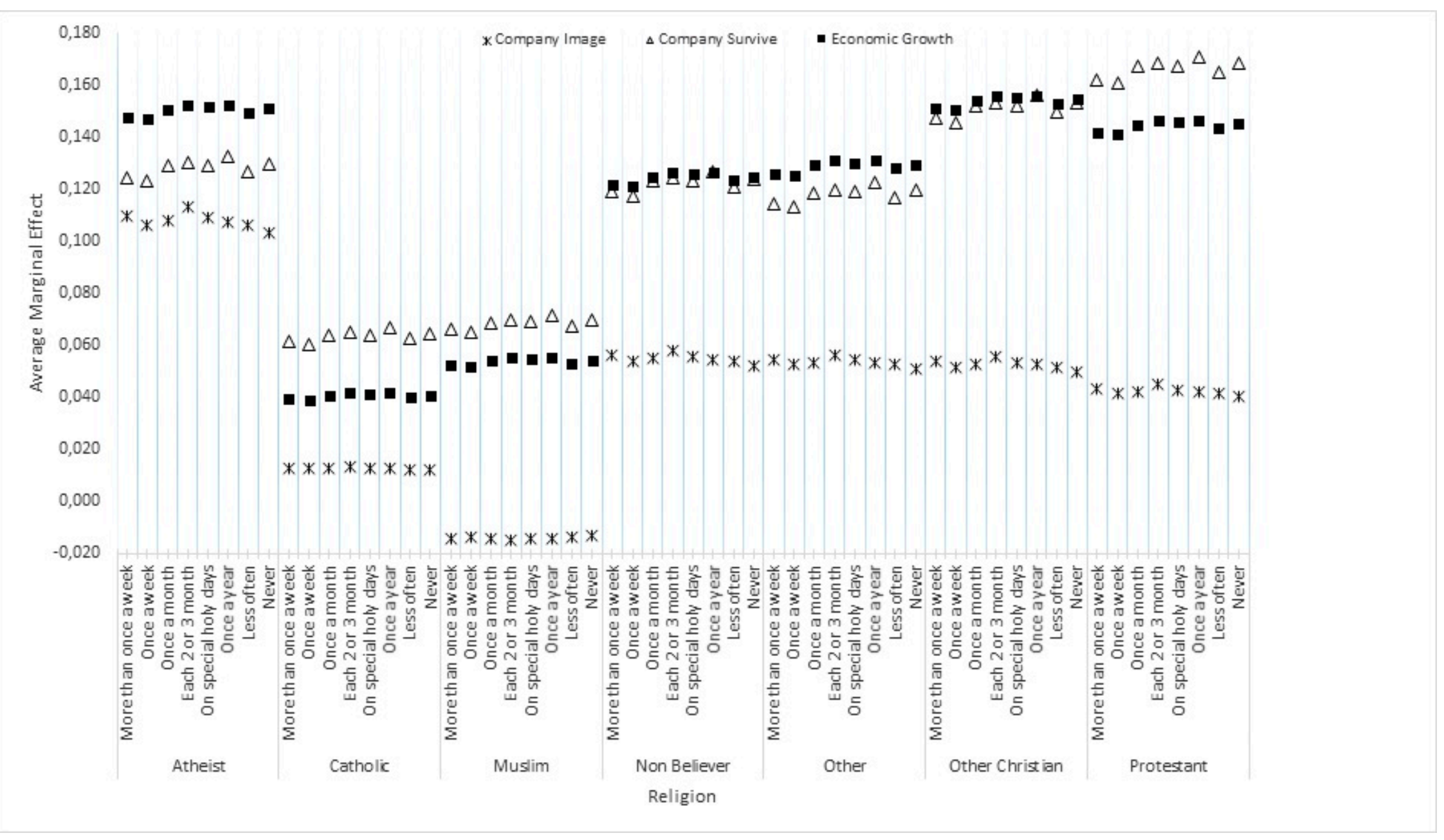

Figure 1. Logit marginal effects. Company image, company survival and economic growth vs. religion/religiosity. 
In short, Europeans' opinion about innovation's outcomes depended on religion denomination. The first group, led by Atheists, Protestants and, at some distance, by other Christians, others and Agnostics, thought that innovation had a strong association with the company's image and capacity to survive, also positively affecting economic growth. Catholics and Muslims made up the second group, characterized by the belief in innovation's modest ability to influence organizations' and economy's results. The Orthodox was the least supportive group as to innovation's capacity of influence.

Furthermore, marginal effects analysis shows that religiosity had not a clear pattern of impact on the dependent variables.

\subsection{Religion and Innovative Product and Service Concept}

Following the methodology explained in the previous section, we found that three different groups emerged (Table 5). The first one was formed of Atheists, Agnostics and Protestants. They were more likely to declare that innovations are mostly gadgets, that innovative products neither are a matter of fashion nor risky to consume and that their advantages are often exaggerated. There was one variable where the probabilities of the three groups did not point in the same direction: Atheists manifested that innovative products simplify everyday life, and Agnostics and Protestants were more likely not to agree with this statement.

The second group was composed of Catholics and others (SPONT.). Their probability to answer that innovations are mainly gadgets was lower than in the previous group and they did not think that innovative products are mainly a fashion. One of their most differentiated characteristics was that they were less likely to report that innovative products simplify everyday life and that innovations are risky for the consumer. Finally, there was a mismatch between these two religion affiliations in relation to the exaggerated communication of the advantages associated to the innovative products: Catholics had a lower probability than the reference group of considering this statement as true, whereas for other (SPONT.), this was just the opposite.

Muslims were quite different from the other groups. They had one of the highest probabilities answering that innovations are mainly gadgets and a matter of fashion, and the lowest odds saying that innovative products simplify life or their advantages are exaggerated. Finally, in spite of the fact that they felt that innovative products are not as risky as the reference category (Orthodox), among the religions group analyzed in this case, they were at the top.

Religion attendance had no significant coefficients for fashion, simplify life and risky. For the other two variables, we found that people who more frequently attended religious acts were more probable to manifest that innovative products are mainly gadgets and their advantages are often exaggerated.

Education also had an effect on the opinion of innovative products. People who stopped their education earlier were more likely to consider that innovative products are a matter of fashion, do not simplify life, are less risky and their advantages are not exaggerated. 
Table 5. Logistic Regression Results on Innovation Concept.

\begin{tabular}{|c|c|c|c|c|c|c|c|c|c|c|c|}
\hline & & \multicolumn{10}{|c|}{ Dependent Variable } \\
\hline & & \multicolumn{2}{|c|}{ Gadgets } & \multicolumn{2}{|c|}{ Fashion } & \multirow{2}{*}{$\begin{array}{c}\text { Simplify Life } \\
\text { Log-Odds }\end{array}$} & \multicolumn{3}{|c|}{ Risky } & \multicolumn{2}{|c|}{ Advantages Exaggerated } \\
\hline & & Log-Odds & Odds & Log-Odds & Odds & & Odds & Log-Odds & Odds & Log-Odds & Odds \\
\hline \multirow[t]{7}{*}{$\begin{array}{c}\text { Religion } \\
\text { (Ref.cat. } \\
\text { Orthodox) }\end{array}$} & Atheist & $\begin{array}{c}0.367^{* * * *} \\
-0.088\end{array}$ & 1.443 & $\begin{array}{c}-0.213 * * * \\
-0.078\end{array}$ & 0.808 & $\begin{array}{l}0.139^{*} \\
-0.071\end{array}$ & 1.149 & $\begin{array}{c}-0.324 * * * \\
-0.093\end{array}$ & 0.724 & $\begin{array}{c}0.410^{* * *} \\
-0.074\end{array}$ & 1.507 \\
\hline & Catholic & $\begin{array}{c}0.188^{* * *} \\
-0.051\end{array}$ & 1.206 & $\begin{array}{c}-0.146^{* * *} \\
-0.042\end{array}$ & 0.864 & $\begin{array}{c}-0.326^{* * *} \\
-0.039\end{array}$ & 0.722 & $\begin{array}{c}-0.485^{* * *} \\
-0.048\end{array}$ & 0.616 & $\begin{array}{l}-0.036 \\
-0.042\end{array}$ & 0.965 \\
\hline & Muslim & $\begin{array}{c}0.346^{* * *} \\
-0.077\end{array}$ & 1.414 & $\begin{array}{l}0.113^{*} \\
-0.065\end{array}$ & 1.120 & $\begin{array}{c}-0.657 * * * \\
-0.065\end{array}$ & 0.518 & $\begin{array}{c}-0.271 * * * \\
-0.079\end{array}$ & 0.763 & $\begin{array}{c}-0.402 * * * \\
-0.074\end{array}$ & 0.669 \\
\hline & Non believer/Agnostic & $\begin{array}{c}0.286^{* * *} \\
-0.071\end{array}$ & 1.331 & $\begin{array}{l}-0.06 \\
-0.06\end{array}$ & 0.942 & $\begin{array}{c}-0.1111^{* *} \\
-0.056\end{array}$ & 0.895 & $\begin{array}{c}-0.446^{* * * *} \\
-0.073\end{array}$ & 0.640 & $\begin{array}{c}0.423 * * * \\
-0.059\end{array}$ & 1.527 \\
\hline & Other (SPONT.) & $\begin{array}{c}0.22 \\
-0.138\end{array}$ & 1.247 & $\begin{array}{c}-0.283 * * \\
-0.123\end{array}$ & 0.754 & $\begin{array}{c}-0.277 * * \\
-0.112\end{array}$ & 0.758 & $\begin{array}{c}-0.372 * * \\
-0.145\end{array}$ & 0.690 & $\begin{array}{c}0.226 * * \\
-0.115\end{array}$ & 1.253 \\
\hline & Other Christian & $\begin{array}{c}0.435^{* * *} \\
-0.087\end{array}$ & 1.545 & $\begin{array}{c}0.103 \\
-0.076\end{array}$ & 1.108 & $\begin{array}{c}0.011 \\
-0.073\end{array}$ & 1.011 & $\begin{array}{l}-0.154^{*} \\
-0.088\end{array}$ & 0.857 & $\begin{array}{c}0.362^{* * *} \\
-0.075\end{array}$ & 1.436 \\
\hline & Protestant & $\begin{array}{c}0.278^{* * *} \\
-0.062\end{array}$ & 1.321 & $\begin{array}{c}0.025 \\
-0.051\end{array}$ & 1.026 & $\begin{array}{c}-0.163 * * * \\
-0.049\end{array}$ & 0.849 & $\begin{array}{c}-0.563 * * * \\
-0.063\end{array}$ & 0.570 & $\begin{array}{c}0.366^{* * *} \\
-0.051\end{array}$ & 1.442 \\
\hline \multirow[t]{7}{*}{$\begin{array}{c}\text { Religiosity } \\
\text { (Ref. cat. More } \\
\text { than once a week) }\end{array}$} & Each 2 or 3 month & $\begin{array}{l}0.197 * * \\
-0.096\end{array}$ & 1.217 & $\begin{array}{l}-0.105 \\
-0.08\end{array}$ & 0.900 & $\begin{array}{l}0.202^{* * * *} \\
'-0.078\end{array}$ & 1.224 & $\begin{array}{l}-0.88 \\
-0.098\end{array}$ & 0.916 & $\begin{array}{l}0.198^{* *} \\
-0.083\end{array}$ & 1.219 \\
\hline & Less often & $\begin{array}{c}-0.99 \\
-0.089\end{array}$ & 1.104 & $\begin{array}{l}-0.097 \\
-0.074\end{array}$ & 0.908 & $\begin{array}{c}0.037 \\
-0.072\end{array}$ & 1.037 & $\begin{array}{c}-0.253 * * * \\
-0.092\end{array}$ & 0.777 & $\begin{array}{l}0.158^{* *} \\
-0.077\end{array}$ & 1.171 \\
\hline & Never & $\begin{array}{l}0.157^{*} \\
-0.086\end{array}$ & 1.170 & $\begin{array}{l}-0.118 \\
-0.072\end{array}$ & 0.889 & $\begin{array}{l}-0.068 \\
-0.071\end{array}$ & 0.934 & $\begin{array}{l}-0.114 \\
-0.088\end{array}$ & 0.892 & $\begin{array}{l}0.146^{*} \\
-0.076\end{array}$ & 1.158 \\
\hline & On special holy days & $\begin{array}{l}0.213 \text { ** } \\
-0.084\end{array}$ & 1.237 & $\begin{array}{c}-0.071 \\
-0.07\end{array}$ & 0.932 & $\begin{array}{c}0.089 \\
-0.069\end{array}$ & 1.093 & $\begin{array}{l}-0.032 \\
-0.085\end{array}$ & 0.969 & $\begin{array}{c}0.216^{* * *} \\
-0.074\end{array}$ & 1.241 \\
\hline & Once a month & $\begin{array}{c}0.183^{* *} \\
-0.092\end{array}$ & 1.201 & $\begin{array}{l}-0.012 \\
-0.077\end{array}$ & 0.988 & $\begin{array}{c}0.155^{* *} \\
-0.075\end{array}$ & 1.168 & $\begin{array}{c}0.015 \\
-0.093\end{array}$ & 1.015 & $\begin{array}{l}0.156^{*} \\
-0.081\end{array}$ & 1.169 \\
\hline & Once a week & $\begin{array}{c}0.265 \text { *** } \\
-0.085\end{array}$ & 1.303 & $\begin{array}{l}-0.003 \\
-0.071\end{array}$ & 0.997 & $\begin{array}{c}0.03 \\
-0.07\end{array}$ & 1.031 & $\begin{array}{c}0.007 \\
-0.086\end{array}$ & 1.007 & $\begin{array}{l}0.158^{* *} \\
-0.075\end{array}$ & 1.171 \\
\hline & Once a year & $\begin{array}{c}0.092 \\
-0.095\end{array}$ & 1.097 & $\begin{array}{l}-0.109 \\
-0.079\end{array}$ & 0.896 & $\begin{array}{c}0.047 \\
-0.077\end{array}$ & 1.048 & $\begin{array}{l}-0.085 \\
-0.097\end{array}$ & 0.919 & $\begin{array}{l}0.173^{* *} \\
-0.082\end{array}$ & 1.188 \\
\hline \multirow[t]{5}{*}{$\begin{array}{l}\text { Marital Status } \\
\text { (Ref. cat. } \\
\text { Widowed) }\end{array}$} & Divorced & $\begin{array}{c}0.113 \\
-0.083\end{array}$ & 1.120 & $\begin{array}{c}0.095 \\
-0.071\end{array}$ & 1.100 & $\begin{array}{l}0.137^{* *} \\
-0.069\end{array}$ & 1.147 & $\begin{array}{c}0.136 \\
-0.086\end{array}$ & 1.146 & $\begin{array}{l}0.141^{* *} \\
-0.071\end{array}$ & 1.151 \\
\hline & Married & $\begin{array}{l}0.142^{* *} \\
-0.057\end{array}$ & 1.153 & $\begin{array}{l}-0.044 \\
-0.05\end{array}$ & 0.957 & $\begin{array}{c}0.244 * * * \\
-0.049\end{array}$ & 1.277 & $\begin{array}{c}0.018 \\
-0.059\end{array}$ & 1.019 & $\begin{array}{c}0.151^{* * *} \\
-0.05\end{array}$ & 1.163 \\
\hline & Other (SPONTANEOUS) & $\begin{array}{c}0.388^{* *} \\
-0.177\end{array}$ & 1.473 & $\begin{array}{c}0.177 \\
-0.154\end{array}$ & 1.194 & $\begin{array}{c}0.229 \\
-0.149\end{array}$ & 1.257 & $\begin{array}{l}-0.051 \\
-0.217\end{array}$ & 0.950 & $\begin{array}{l}-0.066 \\
-0.165\end{array}$ & 0.936 \\
\hline & Remarried & $\begin{array}{c}0.044 \\
-0.119\end{array}$ & 1.045 & $\begin{array}{l}0.075 \\
-0.1\end{array}$ & 1.078 & $\begin{array}{c}0.366 * * * \\
-0.095\end{array}$ & 1.442 & $\begin{array}{c}0.101 \\
-0.121\end{array}$ & 1.106 & $\begin{array}{l}0.184^{*} \\
-0.098\end{array}$ & 1.203 \\
\hline & Separated & $\begin{array}{c}0.410^{* * *} \\
-0.129\end{array}$ & 1.507 & $\begin{array}{c}-0.01 \\
-0.119\end{array}$ & 0.990 & $\begin{array}{c}0.135 \\
-0.114\end{array}$ & 1.145 & $\begin{array}{c}0.262 * \\
-0.14\end{array}$ & 1.300 & $\begin{array}{c}0.024 \\
-0.121\end{array}$ & 1.024 \\
\hline
\end{tabular}


Table 5. Cont

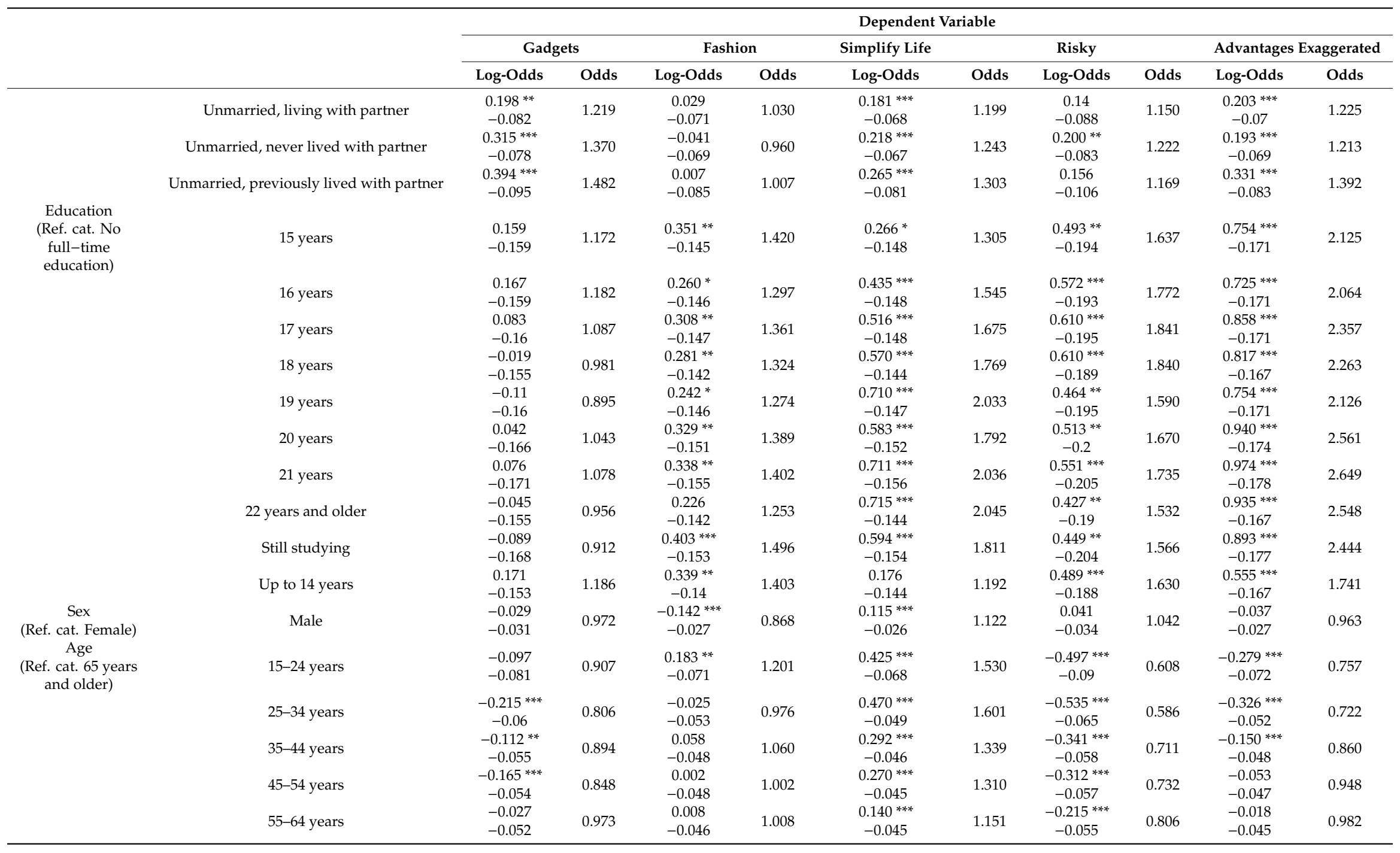


Table 5. Cont.

\begin{tabular}{|c|c|c|c|c|c|c|c|c|c|c|c|}
\hline & & \multicolumn{10}{|c|}{ Dependent Variable } \\
\hline & & \multicolumn{2}{|c|}{ Gadgets } & \multicolumn{2}{|c|}{ Fashion } & \multirow{2}{*}{$\begin{array}{c}\text { Simplify Life } \\
\text { Log-Odds }\end{array}$} & \multicolumn{3}{|c|}{ Risky } & \multicolumn{2}{|c|}{ Advantages Exaggeratec } \\
\hline & & Log-Odds & Odds & Log-Odds & Odds & & Odds & Log-Odds & Odds & Log-Odds & Odds \\
\hline \multirow[t]{6}{*}{$\begin{array}{l}\text { Community } \\
\text { (Ref. cat. Rural } \\
\text { area) }\end{array}$} & Large town & $\begin{array}{c}0.027 \\
-0.039\end{array}$ & 1.028 & $\begin{array}{c}0.024 \\
-0.034\end{array}$ & 1.024 & $\begin{array}{c}0.091^{* * *} \\
-0.032\end{array}$ & 1.095 & $\begin{array}{c}0.037 \\
-0.041\end{array}$ & 1.037 & $\begin{array}{l}-0.045 \\
-0.034\end{array}$ & 0.956 \\
\hline & Small or middle sized town & $\begin{array}{l}-0.019 \\
-0.036\end{array}$ & 0.981 & $\begin{array}{l}-0.008 \\
-0.031\end{array}$ & 0.992 & $\begin{array}{c}0.027 \\
-0.029\end{array}$ & 1.027 & $\begin{array}{c}-0.102 * * * \\
-0.039\end{array}$ & 0.903 & $\begin{array}{c}-0.116 \text { **** } \\
-0.031\end{array}$ & 0.891 \\
\hline & Constant & $\begin{array}{c}-1.883^{* * *} \\
-0.177\end{array}$ & 0.153 & $\begin{array}{c}-0.949^{* * *} \\
-0.157\end{array}$ & 0.387 & $\begin{array}{c}-1.241^{* * *} \\
-0.16\end{array}$ & 0.289 & $\begin{array}{c}-1.439^{* * *} \\
-0.205\end{array}$ & 0.237 & $\begin{array}{c}-1.792 * * * \\
-0.183\end{array}$ & 0.167 \\
\hline & Observations & 28.052 & & 28.052 & & 28.052 & & 28052 & & 28052 & \\
\hline & Log Likelihood & $-13,893.19$ & & $17,091.11$ & & $18,507.56$ & & -12453.93 & & -17211.76 & \\
\hline & Akaike Inf. Crit. & 27.86839 & & 34.26422 & & 37.09711 & & 2498986 & & 3450551 & \\
\hline
\end{tabular}

Note: ${ }^{*} p<0.05 ;{ }^{* *} p<0.01 ;{ }^{* * *} p<0.001$. 


\subsection{Innovative Product and Service Concept Marginal Effects}

Looking at marginal effects (from here onwards, we used the acronym m.e. to report marginal effects results), we see that the comments and relations already found held. Figure 2 exhibits how Europeans feel that innovative products are mainly gadgets. The range of variation was tight, going from m.e. $=2.7 \%$ for Catholics to m.e. $=7 \%$ for other Christians. Religiosity did not affect the results.

We could also see the Europeans' opinion about whether innovative products are a matter of fashion. Results were not significant for Agnostics, other Christians and Protestants (apart from the already known Buddhists, Hindus, Jews and Sikhs). Analysis in this variable must be done in the reversed way for interpretation in innovation terms. It means that those who declared that innovation products are a matter of fashion were less innovative, so the most pro-innovation profile was held by others (SPONT.; m.e. $=-5.7 \%$ ), followed by Atheists (m.e. $=-4.5 \%$ ). Catholics were in the mid-range ranking $($ m.e. $=-3 \%)$. In the opposite situation, Muslims had a worse opinion of innovation products, more so than the Orthodox (the reference category; m.e. $=2.5 \%$ ). Religiosity did not affect the results.

Figure 2 also displays the marginal effects on Europeans' opinion about whether innovative products and services simplify their everyday life. Atheists were the top supporters of this statement (m.e. $=3.3 \%$ ) and Muslims the top detractors (m.e. $=-15 \%$ ). It has to be noted that the sign of the marginal effect was negative, meaning that they had an even worse effect on the dependent variable than the Orthodox (the reference category, chosen for their anti-innovative profile). In the same situation, with negative marginal effects ranked according to the magnitude of importance of them, are Muslims (m.e. $=-15 \%)$, Catholics $(\mathrm{m} . e .=-7.7 \%)$, others $($ SPONT.; m.e. $=-6.6 \%)$, Protestants (m.e. $=-3.9 \%$ ) and Agnostics (m.e. $=-2.6 \%$ ). Religiosity did not affect the results.

Finally, Figure 2 shows the marginal effects of innovative products consumption and the associated risk perception. All the marginal effects had negative signs, meaning that the Orthodox was the religious affiliation that felt more at risk when consuming innovative products and services (reversed scale interpretation). The rest varied between a $2.5 \%$ for other Christians and $8.5 \%$ for Protestants. Religiosity did not have a clear pattern of association. 


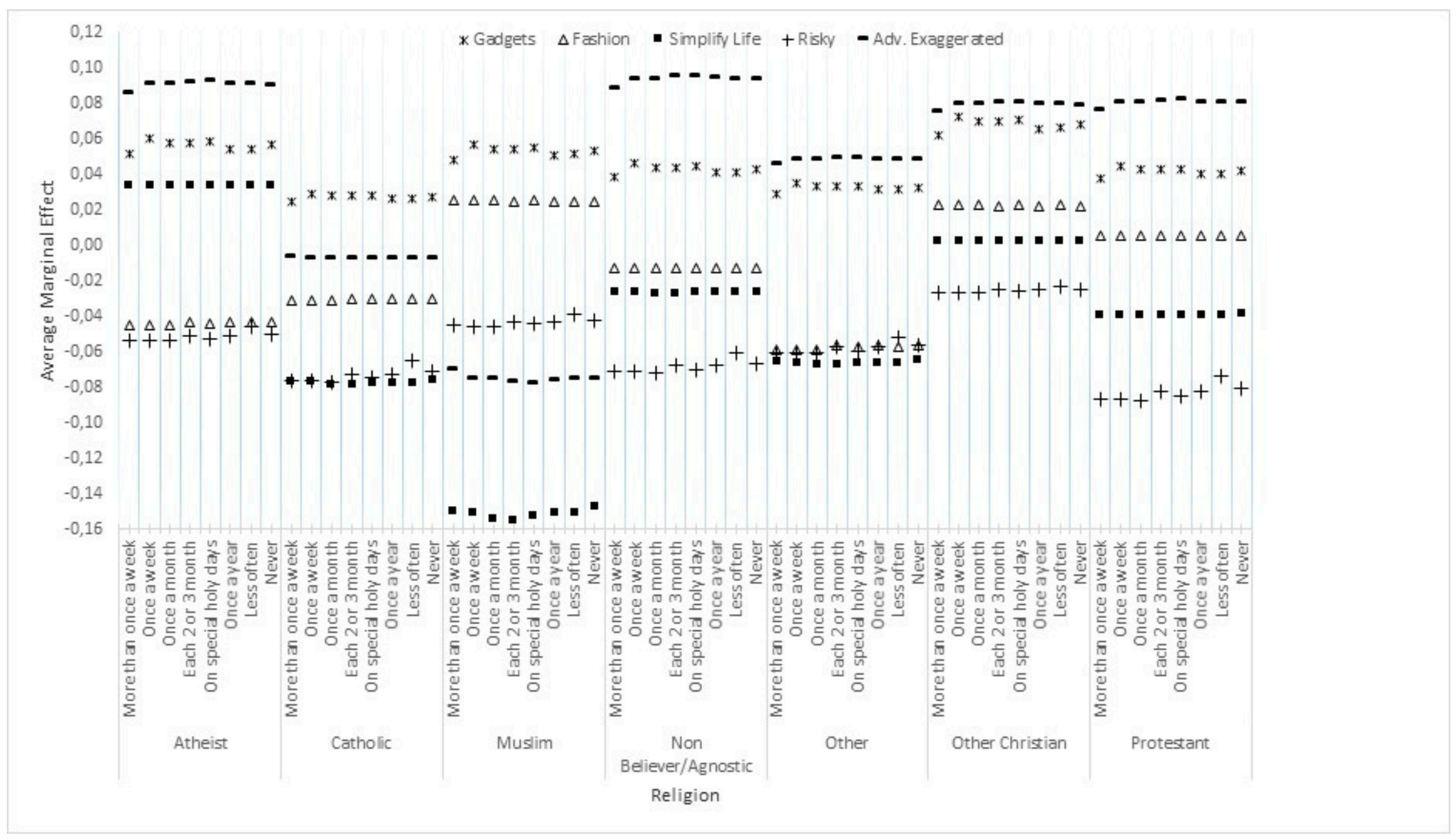

Figure 2. Logit marginal effects. Innovative products as gadgets, fashion, simplify life, risky and advantages exaggerated vs. religion/religiosity. 


\subsection{Attitudes and Purchasing Intentions for Innovative Products}

Coefficients from the logistic regression of attitudes and purchasing behavior of innovative products on religion affiliations show that Muslims had a different and significant profile from the other religion affiliations. They were the most attracted to innovative products (odds $=2.073$ ), and the most likely group to purchase an innovative product that replaces the one that they already trust and regularly buy (odds $=1.420$ ), even if it was more expensive (odds $=1.086$ ). The probability of buying more innovative products than their friends and relatives was also the highest (odds $=1.724$ ). Finally, innovation for them was more associated with the improvement of the existing products than creating new ones (odds $=1.485$; Table 6 ).

We also found that all the other religion affiliations, except Atheists, formed a group because the differences among them were not big. Atheists had a slightly upper pro-innovate behavior and purchasing intentions than the other groups.

Agnostics were one step behind. They declared to be fairly attracted to innovations, more inclined towards innovative products than their friends and relatives, but they only replaced a product that they trusted with an innovation only if the price was a little more expensive.

For Catholics, innovations did not imply the creation of new products but the improvement of existing ones. Should an innovation be introduced in the market, which substitutes an existing product that the Catholics already trust, they would maintain their fidelity with the old product, and they would not pay any more for the new one.

Therefore, religion had an effect on attitudes and purchasing behavior of innovative products.

Most of the coefficients related to religion attendance (religiosity) were not significant for all the dependent variables considered in this section. 
Table 6. Logistic regression results.

\begin{tabular}{|c|c|c|c|c|c|c|c|c|c|c|c|}
\hline & & \multicolumn{10}{|c|}{ Dependent Variable } \\
\hline & & \multicolumn{2}{|c|}{ Gadgets } & \multicolumn{2}{|c|}{ Fashion } & \multicolumn{2}{|c|}{ Simplify Life } & \multicolumn{2}{|c|}{ Risky } & \multicolumn{2}{|c|}{ Advantages Exaggerated } \\
\hline & & Log-Odds & Odds & Log-Odds & Odds & Log-Dds & Odds & Log-Dds & Odds & Log-Odds & Odds \\
\hline \multirow[t]{7}{*}{$\begin{array}{l}\text { Religion } \\
\text { (Ref. cat. } \\
\text { Orthodox) }\end{array}$} & Atheist & $\begin{array}{r}0.368^{* * *} \\
-0.078\end{array}$ & 1.445 & $\begin{array}{r}0.328^{* * *} \\
-0.079\end{array}$ & 1.388 & $\begin{array}{r}0.379 * * * \\
-0.075\end{array}$ & 1.460 & $\begin{array}{l}-0.081 \\
-0.077\end{array}$ & 0.922 & $\begin{array}{r}0.419 * * * \\
-0.076\end{array}$ & 1.520 \\
\hline & Catholic & $\begin{array}{r}0.180^{* * *} \\
-0.042\end{array}$ & 1.197 & $\begin{array}{r}0.049 \\
-0.047\end{array}$ & 1.050 & $\begin{array}{r}0.399 * * * \\
-0.042\end{array}$ & 1.491 & $\begin{array}{r}-0.303^{* * *} \\
-0.043\end{array}$ & 0.739 & $\begin{array}{l}0.093^{* *} \\
-0.044\end{array}$ & 1.097 \\
\hline & Muslim & $\begin{array}{r}0.729 * * * \\
-0.072\end{array}$ & 2.073 & $\begin{array}{r}0.545^{* * *} \\
-0.07\end{array}$ & 1.724 & $\begin{array}{r}0.396 \text { *** } \\
-0.066\end{array}$ & 1.485 & $\begin{array}{r}0.350 * * * \\
-0.07\end{array}$ & 1.420 & $\begin{array}{r}0.082 \\
-0.069\end{array}$ & 1.086 \\
\hline & Non believer/Agnostic & $\begin{array}{c}0.110^{*} \\
-0.06\end{array}$ & 1.116 & $\begin{array}{r}0.071 \\
-0.064\end{array}$ & 1.073 & $\begin{array}{r}0.451^{* * *} \\
-0.059\end{array}$ & 1.569 & $\begin{array}{r}-0.229^{* * *} \\
-0.06\end{array}$ & 0.795 & $\begin{array}{r}0.190 * * * \\
-0.06\end{array}$ & 1.210 \\
\hline & Other (SPONT.) & $\begin{array}{r}0.131 \\
-0.118\end{array}$ & 1.140 & $\begin{array}{r}0.046 \\
-0.127\end{array}$ & 1.048 & $\begin{array}{r}0.448^{* * *} \\
-0.116\end{array}$ & 1.566 & $\begin{array}{l}-0.154 \\
-0.118\end{array}$ & 0.857 & $\begin{array}{r}0.273^{* *} \\
-0.119\end{array}$ & 1.313 \\
\hline & Other Christian & $\begin{array}{l}0.132 * \\
-0.077\end{array}$ & 1.141 & $\begin{array}{r}0.12 \\
-0.085\end{array}$ & 1.128 & $\begin{array}{r}0.427 * * * \\
-0.078\end{array}$ & 1.532 & $\begin{array}{r}-0.274^{* * *} \\
-0.078\end{array}$ & 0.761 & $\begin{array}{r}0.206^{* * * *} \\
-0.078\end{array}$ & 1.228 \\
\hline & Protestant & $\begin{array}{r}0.079 \\
-0.052\end{array}$ & 1.082 & $\begin{array}{l}-0.002 \\
-0.058\end{array}$ & 0.998 & $\begin{array}{r}0.307^{* * *} \\
-0.052\end{array}$ & 1.359 & $\begin{array}{r}-0.304^{* * *} \\
-0.052\end{array}$ & 0.738 & $\begin{array}{r}0.241^{* * * *} \\
-0.053\end{array}$ & 1.272 \\
\hline \multirow[t]{7}{*}{$\begin{array}{l}\text { Religiosity } \\
\text { (Ref. cat. More } \\
\text { than once a week) }\end{array}$} & Each 2 or 3 month & $\begin{array}{l}-0.017 \\
-0.081\end{array}$ & 0.983 & $\begin{array}{l}-0.132 \\
-0.091\end{array}$ & 0.876 & $\begin{array}{r}-0.069 \\
-0.08\end{array}$ & 0.933 & $\begin{array}{r}0.029 \\
-0.081\end{array}$ & 1.030 & $\begin{array}{r}0.093 \\
-0.084\end{array}$ & 1.098 \\
\hline & Less often & $\begin{array}{r}-0.191 * * \\
-0.075\end{array}$ & 0.826 & $\begin{array}{r}-0.193 * * \\
-0.084\end{array}$ & 0.824 & $\begin{array}{r}0.013 \\
-0.074\end{array}$ & 1.013 & $\begin{array}{r}0.072 \\
-0.076\end{array}$ & 1.074 & $\begin{array}{l}-0.077 \\
-0.078\end{array}$ & 0.926 \\
\hline & Never & $\begin{array}{r}-0.212 * * * \\
-0.073\end{array}$ & 0.809 & $\begin{array}{r}-0.155^{*} \\
-0.082\end{array}$ & 0.857 & $\begin{array}{l}-0.069 \\
-0.072\end{array}$ & 0.933 & $\begin{array}{r}0.015 \\
-0.073\end{array}$ & 1.015 & $\begin{array}{r}-0.11 \\
-0.076\end{array}$ & 0.896 \\
\hline & On special holy days & $\begin{array}{c}-0.119 * \\
-0.071\end{array}$ & 0.888 & $\begin{array}{r}-0.137 \text { * } \\
-0.08\end{array}$ & 0.872 & $\begin{array}{r}-0.015 \\
-0.07\end{array}$ & 0.985 & $\begin{array}{r}0.095 \\
-0.072\end{array}$ & 1.100 & $\begin{array}{l}-0.085 \\
-0.074\end{array}$ & 0.919 \\
\hline & Once a month & $\begin{array}{l}-0.044 \\
-0.078\end{array}$ & 0.957 & $\begin{array}{l}-0.142 \\
-0.088\end{array}$ & 0.867 & $\begin{array}{l}-0.011 \\
-0.077\end{array}$ & 0.989 & $\begin{array}{l}-0.111 \\
-0.078\end{array}$ & 0.895 & $\begin{array}{l}-0.039 \\
-0.081\end{array}$ & 0.962 \\
\hline & Once a week & $\begin{array}{l}-0.118 \\
-0.072\end{array}$ & 0.889 & $\begin{array}{r}-0.179 * * \\
-0.082\end{array}$ & 0.836 & $\begin{array}{r}0.055 \\
-0.071\end{array}$ & 1.057 & $\begin{array}{r}-0.209 * * * \\
-0.073\end{array}$ & 0.811 & $\begin{array}{l}-0.051 \\
-0.076\end{array}$ & 0.950 \\
\hline & Once a year & $\begin{array}{r}-0.155^{*} \\
-0.08\end{array}$ & 0.856 & $\begin{array}{r}-0.149 * \\
-0.088\end{array}$ & 0.861 & $\begin{array}{r}0.052 \\
-0.078\end{array}$ & 1.053 & $\begin{array}{l}0.106 \\
-0.08\end{array}$ & 1.112 & $\begin{array}{l}-0.098 \\
-0.082\end{array}$ & 0.907 \\
\hline \multirow[t]{5}{*}{$\begin{array}{l}\text { Marital Status } \\
\text { (Ref. cat. } \\
\text { Widowed) }\end{array}$} & Divorced & $\begin{array}{c}0.123 * \\
-0.07\end{array}$ & 1.131 & $\begin{array}{l}0.162 * \\
-0.088\end{array}$ & 1.176 & $\begin{array}{r}0.053 \\
-0.071\end{array}$ & 1.054 & $\begin{array}{r}0.215 \text { *** } \\
-0.072\end{array}$ & 1.240 & $\begin{array}{l}0.161 \text { ** } \\
-0.077\end{array}$ & 1.175 \\
\hline & Married & $\begin{array}{r}0.294 * * * \\
-0.05\end{array}$ & 1.342 & $\begin{array}{r}0.224 * * * \\
-0.067\end{array}$ & 1.251 & $\begin{array}{l}-0.048 \\
-0.051\end{array}$ & 0.953 & $\begin{array}{r}0.289 * * * \\
-0.052\end{array}$ & 1.336 & $\begin{array}{r}0.363 * * * \\
-0.057\end{array}$ & 1.438 \\
\hline & Other (SPONTANEOUS) & $\begin{array}{c}0.368^{* *} \\
-0.173\end{array}$ & 1.445 & $\begin{array}{r}0.134 \\
-0.166\end{array}$ & 1.143 & $\begin{array}{l}-0.142 \\
-0.155\end{array}$ & 0.867 & $\begin{array}{r}0.122 \\
-0.156\end{array}$ & 1.129 & $\begin{array}{r}0.521^{* * *} \\
-0.157\end{array}$ & 1.684 \\
\hline & Remarried & $\begin{array}{r}0.463 * * * \\
-0.1\end{array}$ & 1.589 & $\begin{array}{r}0.413 * * * \\
-0.113\end{array}$ & 1.511 & $\begin{array}{r}0.015 \\
-0.099\end{array}$ & 1.015 & $\begin{array}{r}0.435^{* * *} \\
-0.099\end{array}$ & 1.544 & $\begin{array}{r}0.504 * * * \\
-0.103\end{array}$ & 1.656 \\
\hline & Separated & $\begin{array}{r}0.166 \\
-0.116\end{array}$ & 1.180 & $\begin{array}{r}0.398 * * * \\
-0.131\end{array}$ & 1.489 & $\begin{array}{l}-0.117 \\
-0.118\end{array}$ & 0.890 & $\begin{array}{r}0.298^{* *} \\
-0.118\end{array}$ & 1.347 & $\begin{array}{r}0.181 \\
-0.124\end{array}$ & 1.198 \\
\hline
\end{tabular}


Table 6. Cont

\begin{tabular}{|c|c|c|c|c|c|c|c|c|c|c|c|}
\hline & & \multicolumn{10}{|c|}{ Dependent Variable } \\
\hline & & \multicolumn{2}{|c|}{ Gadgets } & \multicolumn{2}{|c|}{ Fashion } & \multicolumn{2}{|c|}{ Simplify Life } & \multicolumn{2}{|c|}{ Risky } & \multicolumn{2}{|c|}{ Advantages Exaggerated } \\
\hline & & Log-Odds & Odds & Log-Odds & Odds & Log-Dds & Odds & Log-Dds & Odds & Log-Odds & Odds \\
\hline \multirow{8}{*}{$\begin{array}{l}\text { Education } \\
\text { (Ref. cat. No } \\
\text { full-time } \\
\text { education) }\end{array}$} & Unmarried, living with partner & $\begin{array}{l}0.168^{* *} \\
-0.071\end{array}$ & 1.183 & $\begin{array}{l}0.142 * \\
-0.084\end{array}$ & 1.152 & $\begin{array}{l}0.008 \\
-0.07\end{array}$ & 1.008 & $\begin{array}{r}0.387^{* * * *} \\
-0.072\end{array}$ & 1.472 & $\begin{array}{r}0.449^{* * *} \\
-0.075\end{array}$ & 1.566 \\
\hline & Unmarried, never lived with partner & $\begin{array}{l}0.176^{* *} \\
-0.069\end{array}$ & 1.192 & $\begin{array}{r}0.231^{* * *} \\
-0.083\end{array}$ & 1.260 & $\begin{array}{r}-0.137^{* *} \\
-0.069\end{array}$ & 0.872 & $\begin{array}{r}0.179^{* *} \\
-0.07\end{array}$ & 1.196 & $\begin{array}{r}0.352 * * * * \\
-0.074\end{array}$ & 1.421 \\
\hline & Unmarried, previously lived with partner & $\begin{array}{l}0.178^{* *} \\
-0.086\end{array}$ & 1.195 & $\begin{array}{r}0.052 \\
-0.098\end{array}$ & 1.054 & $\begin{array}{r}0.093 \\
-0.084\end{array}$ & 1.097 & $\begin{array}{r}0.096 \\
-0.086\end{array}$ & 1.100 & $\begin{array}{r}0.305^{* * * *} \\
-0.088\end{array}$ & 1.357 \\
\hline & 15 years & $\begin{array}{r}0.092 \\
-0.144\end{array}$ & 1.096 & $\begin{array}{r}0.127 \\
-0.188\end{array}$ & 1.136 & $\begin{array}{r}0.116 \\
-0.154\end{array}$ & 1.123 & $\begin{array}{l}0.294^{*} \\
-0.155\end{array}$ & 1.342 & $\begin{array}{l}-0.247 \\
-0.159\end{array}$ & 0.781 \\
\hline & 16 years & $\begin{array}{c}0.353 * * \\
-0.143\end{array}$ & 1.424 & $\begin{array}{c}0.455^{* *} \\
-0.185\end{array}$ & 1.576 & $\begin{array}{l}-0.096 \\
-0.153\end{array}$ & 0.909 & $\begin{array}{r}0.507^{* * * *} \\
-0.155\end{array}$ & 1.660 & $\begin{array}{r}0.08 \\
-0.158\end{array}$ & 1.084 \\
\hline & 17 years & $\begin{array}{c}0.343^{* *} \\
-0.144\end{array}$ & 1.410 & $\begin{array}{c}0.379 * * \\
-0.186\end{array}$ & 1.460 & $\begin{array}{l}-0.029 \\
-0.154\end{array}$ & 0.972 & $\begin{array}{r}0.516^{* * *} \\
-0.156\end{array}$ & 1.675 & $\begin{array}{r}0.116 \\
-0.158\end{array}$ & 1.123 \\
\hline & 18 years & $\begin{array}{r}0.486 * * * \\
-0.14\end{array}$ & 1.626 & $\begin{array}{c}0.399 * * \\
-0.182\end{array}$ & 1.491 & $\begin{array}{r}0.02 \\
-0.15\end{array}$ & 1.021 & $\begin{array}{r}0.614^{* * * *} \\
-0.151\end{array}$ & 1.847 & $\begin{array}{r}0.234 \\
-0.154\end{array}$ & 1.264 \\
\hline & 19 years & $\begin{array}{r}0.764^{* * *} \\
-0.144\end{array}$ & 2.148 & $\begin{array}{r}0.579 * * * \\
-0.185\end{array}$ & 1.784 & $\begin{array}{r}0.057 \\
-0.153\end{array}$ & 1.059 & $\begin{array}{r}0.777^{* * *} \\
-0.155\end{array}$ & 2.175 & $\begin{array}{r}0.407 * * * \\
-0.157\end{array}$ & 1.503 \\
\hline \multirow{11}{*}{$\begin{array}{l}\text { Sex } \\
\text { (Ref. cat. Female) } \\
\text { Age } \\
\text { (Ref. cat. } 65 \text { years } \\
\text { and older) }\end{array}$} & 20 years & $\begin{array}{r}0.773 * * * \\
-0.149\end{array}$ & 2.166 & $\begin{array}{r}0.567 * * * \\
-0.189\end{array}$ & 1.762 & $\begin{array}{l}-0.097 \\
-0.158\end{array}$ & 0.907 & $\begin{array}{r}0.732 * * * \\
-0.16\end{array}$ & 2.080 & $\begin{array}{r}0.416^{* * * *} \\
-0.161\end{array}$ & 1.515 \\
\hline & 21 years & $\begin{array}{c}0.794^{* * *} \\
-0.154\end{array}$ & 2.212 & $\begin{array}{r}0.531^{* * *} \\
-0.193\end{array}$ & 1.701 & $\begin{array}{l}-0.115 \\
-0.161\end{array}$ & 0.891 & $\begin{array}{r}0.644^{* * * *} \\
-0.163\end{array}$ & 1.904 & $\begin{array}{r}0.471^{* * * *} \\
-0.165\end{array}$ & 1.601 \\
\hline & 22 years and older & $\begin{array}{r}0.873^{* * *} \\
-0.14\end{array}$ & 2.393 & $\begin{array}{r}0.687^{* * *} \\
-0.181\end{array}$ & 1.989 & $\begin{array}{r}-0.270^{*} \\
-0.15\end{array}$ & 0.764 & $\begin{array}{r}0.815^{* * * *} \\
-0.152\end{array}$ & 2.259 & $\begin{array}{r}0.658^{* * * *} \\
-0.154\end{array}$ & 1.932 \\
\hline & Still studying & $\begin{array}{r}0.844^{* * *} \\
-0.154\end{array}$ & 2.325 & $\begin{array}{r}0.560^{* * *} \\
-0.189\end{array}$ & 1.751 & $\begin{array}{r}-0.114 \\
-0.16\end{array}$ & 0.892 & $\begin{array}{r}0.764^{* * * *} \\
-0.162\end{array}$ & 2.147 & $\begin{array}{r}0.374 * * \\
-0.163\end{array}$ & 1.453 \\
\hline & Up to 14 years & $\begin{array}{r}0.028 \\
-0.139\end{array}$ & 1.028 & $\begin{array}{l}-0.026 \\
-0.182\end{array}$ & 0.974 & $\begin{array}{r}-0.1 \\
-0.149\end{array}$ & 0.905 & $\begin{array}{r}0.19 \\
-0.151\end{array}$ & 1.209 & $\begin{array}{r}-0.374^{* *} \\
-0.154\end{array}$ & 0.688 \\
\hline & Male & $\begin{array}{r}0.308^{* * *} \\
-0.027\end{array}$ & 1.360 & $\begin{array}{r}0.334^{* * *} \\
-0.029\end{array}$ & 1.397 & $\begin{array}{r}-0.065 * * \\
-0.027\end{array}$ & 0.937 & $\begin{array}{l}0.070^{* *} \\
-0.027\end{array}$ & 1.072 & $\begin{array}{r}0.226^{* * * *} \\
-0.027\end{array}$ & 1.254 \\
\hline & $15-24$ years & $\begin{array}{r}1.298^{* * *} \\
-0.074\end{array}$ & 3.660 & $\begin{array}{r}1.125 * * * \\
-0.077\end{array}$ & 3.079 & $\begin{array}{r}-0.156 * * \\
-0.07\end{array}$ & 0.856 & $\begin{array}{r}1.106^{* * *} \\
-0.072\end{array}$ & 3.021 & $\begin{array}{r}0.843 * * * * \\
-0.072\end{array}$ & 2.323 \\
\hline & $25-34$ years & $\begin{array}{r}1.059^{* * *} \\
-0.052\end{array}$ & 2.885 & $\begin{array}{r}0.915^{* * *} \\
-0.059\end{array}$ & 2.498 & $\begin{array}{r}-0.230 * * * \\
-0.051\end{array}$ & 0.794 & $\begin{array}{r}0.909 * * * \\
-0.052\end{array}$ & 2.482 & $\begin{array}{r}0.665^{* * * *} \\
-0.053\end{array}$ & 1.945 \\
\hline & $35-44$ years & $\begin{array}{c}0.835^{* * *} \\
-0.047\end{array}$ & 2.304 & $\begin{array}{r}0.709^{* * *} \\
-0.056\end{array}$ & 2.032 & $\begin{array}{r}-0.260^{* * *} \\
-0.047\end{array}$ & 0.771 & $\begin{array}{r}0.785^{* * * *} \\
-0.048\end{array}$ & 2.193 & $\begin{array}{r}0.634^{* * * *} \\
-0.05\end{array}$ & 1.885 \\
\hline & $45-54$ years & $\begin{array}{r}0.572 * * * \\
-0.046\end{array}$ & 1.772 & $\begin{array}{r}0.406^{* * *} \\
-0.057\end{array}$ & 1.501 & $\begin{array}{r}-0.156^{* * *} \\
-0.047\end{array}$ & 0.855 & $\begin{array}{r}0.534^{* * * *} \\
-0.047\end{array}$ & 1.706 & $\begin{array}{r}0.469 * * * * \\
-0.05\end{array}$ & 1.598 \\
\hline & $55-64$ years & $\begin{array}{r}0.376 * * * \\
-0.045\end{array}$ & 1.457 & $\begin{array}{r}0.359^{* * *} \\
-0.057\end{array}$ & 1.432 & $\begin{array}{r}-0.101 * * \\
-0.046\end{array}$ & 0.904 & $\begin{array}{r}0.347^{* * *} \\
-0.046\end{array}$ & 1.414 & $\begin{array}{r}0.342 * * * \\
-0.049\end{array}$ & 1.407 \\
\hline
\end{tabular}


Table 6. Cont.

\begin{tabular}{|c|c|c|c|c|c|c|c|c|c|c|c|}
\hline & & \multicolumn{10}{|c|}{ Dependent Variable } \\
\hline & & \multicolumn{2}{|c|}{ Gadgets } & \multicolumn{2}{|c|}{ Fashion } & \multicolumn{2}{|c|}{ Simplify Life } & \multicolumn{2}{|c|}{ Risky } & \multicolumn{2}{|c|}{ Advantages Exaggerated } \\
\hline & & Log-Odds & Odds & Log-Odds & Odds & Log-Dds & Odds & Log-Dds & Odds & Log-Odds & Odds \\
\hline \multirow[t]{6}{*}{$\begin{array}{l}\text { Community } \\
\text { (Ref. cat. } \\
\text { Rural area) }\end{array}$} & Large town & $\begin{array}{r}0.120^{* * *} \\
-0.034\end{array}$ & 1.127 & $\begin{array}{r}0.140^{* * *} \\
-0.037\end{array}$ & 1.151 & $\begin{array}{r}-0.057^{*} \\
-0.033\end{array}$ & 0.95 & $\begin{array}{l}0.081 * * \\
-0.034\end{array}$ & 1.085 & $\begin{array}{l}0.085 * * \\
-0.034\end{array}$ & 1.088 \\
\hline & Small or middle sized town & $\begin{array}{c}0.107^{* * *} \\
-0.031\end{array}$ & 1.113 & $\begin{array}{l}0.069 * * \\
-0.034\end{array}$ & 1.072 & $\begin{array}{r}0.006 \\
-0.03\end{array}$ & 1.01 & $\begin{array}{r}0.105^{* * * *} \\
-0.031\end{array}$ & 1.111 & $\begin{array}{r}0.135 * * * * \\
-0.031\end{array}$ & 1.144 \\
\hline & Constant & $\begin{array}{c}-1.238 * * * \\
-0.157\end{array}$ & 0.290 & $\begin{array}{r}-2.306 * * * \\
-0.203\end{array}$ & 0.1 & $\begin{array}{c}-0.289 * \\
-0.166\end{array}$ & 0.75 & $\begin{array}{r}-1.189^{* * *} \\
-0.168\end{array}$ & 0.305 & $\begin{array}{r}-1.686^{* * *} \\
-0.172\end{array}$ & 0.185 \\
\hline & Observations & 26.212 & & 26.212 & & 25.298 & & 25.817 & & 25.96 & \\
\hline & Log Likelihood & $-14,830.99$ & & $-14,830.99$ & & $-17,155.17$ & & $-16,810.79$ & & $-16,533.69$ & \\
\hline & Akaike Inf. Crit. & 29.74399 & & 29.74399 & & 34.39235 & & 33.70357 & & 33.14938 & \\
\hline
\end{tabular}

Note: ${ }^{*} p<0.05 ;{ }^{* *} p<0.01$; ${ }^{* * *} p<0.001$. 


\subsection{Attitudes and Purchasing Intentions for Innovative Products Marginal Effects}

Figure 3 shows that Muslims (m.e. 15\%) played the leading position in being attracted by innovations, followed by Atheists (m.e. 8\%). Catholics, Agnostics, other Christians and Protestants form a group that declared not to be very attracted to innovation (m.e. between $2.5 \%$ and $4 \%$ ). Religiosity did not affect the results.

Muslims and Atheists were the only studied affiliations that had significant marginal effects on purchasing intentions of innovative products. Muslims were more inclined (m.e. $=11 \%$ ) to purchase innovative products than the reference category (Orthodox). Atheists (m.e. $=6.5 \%$ ) stood in the middle range between both. Religiosity did not affect the results.

Table 3 also presents the Europeans' opinion whether innovation means the creation of new products or the improvement of existing ones. This information should be interpreted as a reversed scale, meaning that the most strict position regarding innovation would be that only the creation of new products could be considered as "true" innovation. This explains why marginal effects appeared with negative signs: The most narrow position, represented by the Orthodox, had an m.e. $=0 \%$, while all the rest presented negative marginal effects (considering that innovations were also improvements made to the products that already existed). A group formed by Atheists, Catholics, Muslims, Agnostics, other Christians and others (SPONT.) also declared sharing a softer approach to innovation (m.e. about $-10 \%)$. Protestants were situated in between this group and the Orthodox, with m.e. $=-7 \%$. Religiosity did not affect the results.

The replacement variable depicts the respondents' opinion about how quickly they would try an innovation when it comes to the market. Catholics, Agnostics, other Christians and Protestants seemed to be moderate first adopters of innovative products and services (m.e. about $4 \%$ to $7 \%$ ). An extreme case appeared, Muslims, showing a top first adopter profile (m.e. $=15 \%$ ).

The last variable studied in this section resumed the impact that price has on Europeans' innovative products purchasing intention. Atheists were the ones who were willing to pay more for innovations $(\mathrm{m}$. e. $=9.5 \%)$. A group formed by Agnostics, other Christians, Protestants and others (SPONT.) were in the middle range (m.e. from $4 \%$ to $6 \%$ ). Finally, Catholics were in the low range $(\mathrm{m} . e .=2 \%$ ). Religiosity did not affect the results. 


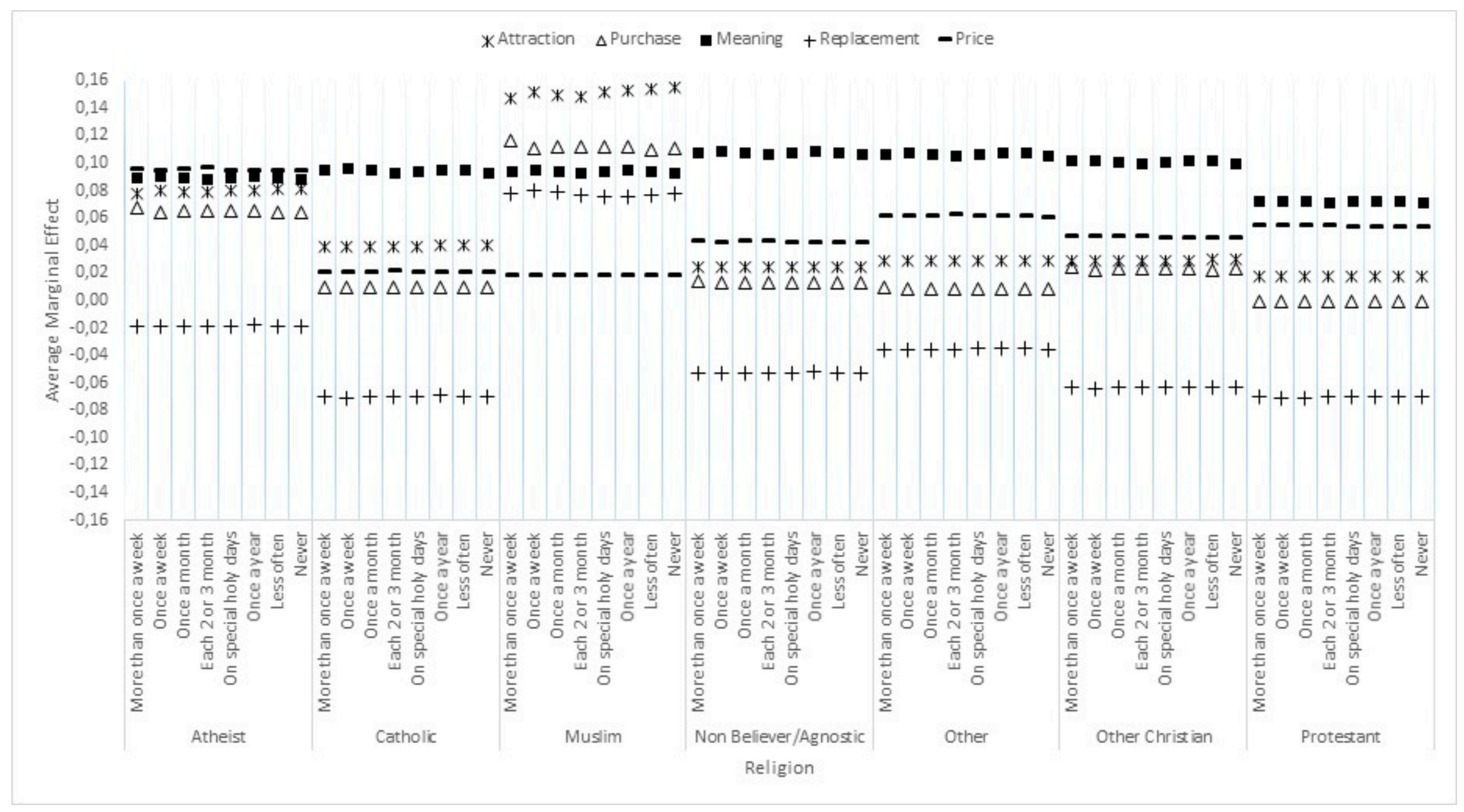

Figure 3. Logit marginal effects. Attitudes and purchasing intentions for innovative products vs. religion/religiosity. 
To summarize, the declared religion was associated with the Europeans' innovative products purchasing behavior. Muslims had the most pro-innovation purchasing intentions. Atheists were very attracted to innovative products and they did not mind if they had to pay more for them. Catholics were not very attracted to innovative products, albeit accepting they were not only the creation of novelties but also the improvement of the existing ones. However, they were only willing to try them if they did not have to pay more. Agnostics, Protestants, other Christians and others (SPONT.) formed a group characterized for sharing a mid-range, pro-innovative products purchasing intention. Finally, religiosity did not have any remarkable association with attitudes and purchasing intentions for innovative products.

\section{Discussion, Implications and Conclusions}

Results obtained from logistic regression confirmed that religion declared affiliation by Europeans affected their innovativeness profile in the major religious faiths present in Europe. Therefore, hypothesis 2 held.

Atheists were the most innovativeness-oriented group. We labeled them as innovation top supporters due to results obtained from the logistic regression odds and marginal effects. Moreover, they also had a good forecast, increasing their participation of the total projected population from $18.8 \%$ in 2010 to $23.3 \%$ in 2050 (Table 7). One would expected Europe to continue its leading position in having unaffiliated people in comparison, for instance, in comparison to the U.S.A. (McBride 2010).

Table 7. Size and projected growth of major religious groups in Europe, 2010-2050.

\begin{tabular}{|c|c|c|c|c|c|c|c|}
\hline & $\begin{array}{l}\quad 2010 \\
\text { Estimated } \\
\text { Population }\end{array}$ & $\%$ in 2010 & $\begin{array}{c}2050 \\
\text { Projected } \\
\text { Population }\end{array}$ & $\%$ in 2050 & $\begin{array}{l}\text { Popultation } \\
\text { Growth } \\
\text { 2010-2050 }\end{array}$ & $\begin{array}{c}\% \\
\text { Increase } \\
2010-2050\end{array}$ & $\begin{array}{l}\text { Compound } \\
\text { Annual } \\
\text { Growth } \\
\text { Rate (\%) }\end{array}$ \\
\hline Christians & $553,280,000$ & 74.5 & $454,090,000$ & 65.2 & $-99,190,000$ & -17.9 & -0.5 \\
\hline Unafiliated & $139,890,000$ & 18.8 & $162,320,000$ & 23.3 & $22,420,000$ & 16 & 0.4 \\
\hline Muslims & $43,470,000$ & 5.9 & $70,870,000$ & 10.2 & $27,400,000$ & 63 & 1.2 \\
\hline Jews & $1,420,000$ & 0.2 & $1,200,000$ & 0.2 & $-220,000$ & -15.2 & -0.4 \\
\hline Hindus & $1,380,000$ & 0.2 & $2,660,000$ & 0.4 & $1,280,000$ & 92.9 & 1.7 \\
\hline Buddhists & $1,350,000$ & 0.2 & $2,490,000$ & 0.4 & $1,140,000$ & 85 & 1.5 \\
\hline $\begin{array}{c}\text { Other } \\
\text { Religions }\end{array}$ & 890,000 & 0.1 & $1,100,000$ & 0.2 & 210,000 & 23.3 & 0.5 \\
\hline $\begin{array}{c}\text { Folk } \\
\text { Religions }\end{array}$ & 870,000 & 0.1 & $1,590,000$ & 0.2 & 720,000 & 83.1 & 1.5 \\
\hline $\begin{array}{l}\text { Regional } \\
\text { Total }\end{array}$ & $742,550,000$ & 100 & $696,330,000$ & 100 & $-46,220,000$ & -6.2 & -0.2 \\
\hline
\end{tabular}

Source: Pew Research Center Religion and Public Life (2015).

Muslims would also increase their participation in the European religion landscape, from $5.9 \%$ in 2010 to $10.2 \%$ in 2050. In addition, the innovativeness profile obtained from our analysis confirmed that they have an open, flexible and adaptative concept of how they perceive innovation and the associated innovative products and services. We labeled them as incremental innovation supporters. For them, innovations embedded in everyday products and services were the path for reducing the inequalities caused by technical progress. It was a market approach to obtain the results that historically were reached through public goods provision from religious institutions. As Michalopoulos et al. (2016) said, Islam restrained inequality in order to survive. Inequality has been one of the consequences of the globalization process that we have been living in the past decades. Europe has also suffered these results (Milanovic 2012). Muslims thought that innovation is good for neither company survival nor economic growth. Therefore, for them, incremental innovations applied to everyday products was a route to reduce inequalities caused by technical and economic progress. Their members obtained advantages not only through the religious nets, but also via market exchange, relaxing the doctrine about consuming innovative products and services in an evolutioned Club Goods model. 
Christians are, and will be, the largest religious group in Europe. Nevertheless, they will reduce their share in European population terms from $74.5 \%$ in 2010 to $65.2 \%$ in 2050 (Table 7). We have worked with the four Christian traditions (Catholic, Protestant, Orthodox and other Christian) that represented the figures shown in Table 8.

Table 8. Christian traditions in Europe and in the world (2010).

\begin{tabular}{ccccc}
\hline & $\begin{array}{c}\text { Estimated 2010 } \\
\text { European } \\
\text { Poulation }\end{array}$ & $\begin{array}{c}\text { Percentage of } \\
\text { European } \\
\text { Christian }\end{array}$ & $\begin{array}{c}\text { Estimated 2010 } \\
\text { World Total }\end{array}$ & $\begin{array}{c}\text { Percentage of } \\
\text { World Total }\end{array}$ \\
\hline Catholic & $254,441,790$ & 55.7 & $1,064,610,000$ & 23.9 \\
Protestant & $100,880,640$ & 22.1 & $800,640,000$ & 12.6 \\
Orthodox & $98,782,220$ & 21.6 & $260,380,000$ & 76.9 \\
Other Christians & $3,098,870$ & 0.7 & $28,430,000$ & 10.9 \\
\hline
\end{tabular}

Source: Hackett et al. (2012).

Catholic is the most important Christian tradition in Europe. In 2010, they represented almost more than 254 million people and 55.7\% of the European Christians. We labeled their innovativeness profile as innovation disbelievers. None of the innovation outcomes-company image, company survival or economic growth - could be easily reached from their viewpoint. All the other variables accomplished-except perceived risk, meaning and buying—also describe anti-innovativeness modes. This implies a big challenge for European authorities and innovative companies in Europe.

The Orthodox' profile was even worse than the Catholic's. They represented almost 99 million Europeans in 2010 and $21.6 \%$ of the European Christians. We labeled them as innovation detractors because the risks associated with innovation inhibit their purchasing behavior and the benefits that would be associated to companies and economic growth.

Protestant was the second most important Christian tradition in Europe that accounted for almost 101 million Europeans in 2010 and 22.1\% of the European Christian. Labeled as stingy innovators, they manifest that innovation is important for companies and economic growth but they are not very inclined to change the products they trust and they would only do so if they have to pay slightly more.

Other Christians represented only $0.7 \%$ of the European Christians in 2010. Their innovative profile was much closer to the Protestants'. The main differences were that they feel that consuming innovative products and services is risky and that innovative products are mainly gadgets. That was why we labeled them as low-profiled stingy innovators.

Finally, Agnostics belonged to the category of unaffiliated in Table 7. Their innovativeness profile, obtained from the study of the logistic regression's marginal effects for religion denomination and religion attendance, is also closely related to the Protestants' and other Christians'. We concluded that they were a step down in the innovativeness ladder because they were price-conscious about purchasing innovative products. We labeled them as price-conscious innovators. Nevertheless, the profile projected in Figure 4 was practically indistinguishable from the Protestants'. Agnostics, Protestants and other Christians were the price-conscious consumers of innovative products and services. 


\begin{tabular}{|c|c|c|c|c|c|c|c|c|c|c|c|c|c|c|}
\hline & \multicolumn{3}{|c|}{ INNOVATION OUTCOMES } & \multicolumn{5}{|c|}{ INNOVATION CONCEPT } & \multicolumn{5}{|c|}{ ATTITUDES AND PURCHASING INTENTIONS } & \\
\hline & $\begin{array}{c}\text { Company } \\
\text { Image }\end{array}$ & $\begin{array}{c}\text { Company } \\
\text { Survival }\end{array}$ & $\begin{array}{l}\text { Economic } \\
\text { Growth }\end{array}$ & Gadgets & Fashion & Simplify Life & Risk & Adv. Exagg. & Attraction & Purchase & Meaning & Buying & Price & \\
\hline Atheist & & & & & & & & & & & & & & \\
\hline Catholic & & & & & & & & & & & & & & Most Innovative \\
\hline Muslim & & & & & & & & & & & & & & More Innovative \\
\hline Ion believer/Agnostic & & & & & & & & & & & & & & Less Innovative \\
\hline Other (SPONT.) & & & & & & & & & & & & & & Least Innovative \\
\hline Other Christian & & & & & & & & & & & & & & Not significant \\
\hline Protestant & & & & & & & & & & & & & & \\
\hline
\end{tabular}

Figure 4. Marginal effects results on all innovation variables under study. 
Our analysis shows that religiosity did not seem to have a very strong relationship with attitudes towards innovation. Hence, our first hypothesis, which says that there is a negative relationship among religiosity and innovation, did not hold. It was obtained from our marginal effects study and, in some way, contradicted recent research made by Bénabou et al. $(2013,2015)$.

While looking for an explanation of the differences among the methodologies used in these publications and the one used in our study, we found that religiosity it was not equally measured. Bénabou et al. (2013), used two measures of religiosity corresponding respectively to the answers to the World Values Survey (WVS) questions: (i) "Independently of whether you go to church or not, would you say you are: a religious person, not a religious person, a convinced atheist, don't know", and: (ii) "Do you believe in God?-Yes, No, Don't Know". We used the one contained in the Eurobarometer that asks about the interviewee religion attendance. If it is intended to measure how the intensity of religion beliefs affects innovative perceptions, intentions and purchasing behavior, we thought that religion attendance was a better proxy than the two WVS questions. Furthermore, innovation is not measured in the same way: they used (log-) patents per capita at country level taken from World Intellectual Property Organization (WIPO). We used the fifteen variables contained in the Eurobarometer. Their only independent variable is a measure on innovation at a macro level, and the one presented in this work is more related to a micro/individual level. In any case, a deeper analysis is needed in order to see how the relationship between innovation/innovativeness and religiosity works.

Innovative companies could take advantage from the outcomes of this paper, by segmenting the market through the religion profiles of their customers. Innovations/technology life-cycle is based on the fact that customers do not accept (and buy) innovations and innovative products at the same time. Following Rogers (1995), it is known that first come the innovators and early adopters (concerned with the underlying technology and its performance). Afterwards, the early majority pragmatists, the late majority conservatives and lastly the laggards (much more interested in solutions and convenience) come onto scene. It has a strong relationship with the innovativeness religion profiles that we have already described. The link can be graphically seen in the next Figure 5.

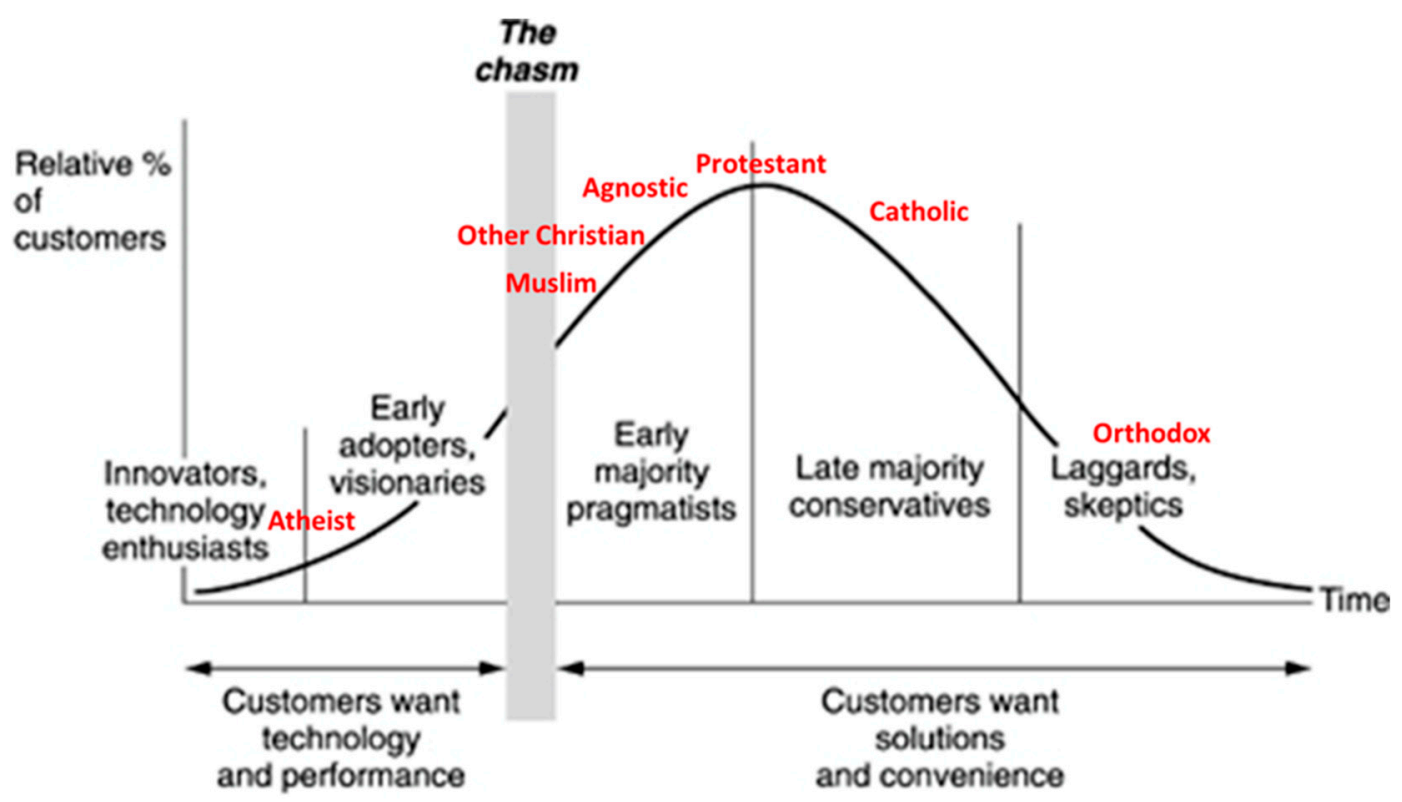

Figure 5. Life cycle and religions in Europe. Source: own elaboration based on Norman (1998).

Results also had some implications for European Union institutions. It would be interesting to have regular ad-hoc information about religion and innovation from the Europeans collected, for instance, via Eurobarometer. The European Commission devotes a lot of effort and money in order to be one of the most innovative regions of the world. However, this effort it is not well connected with the information collected from primary sources on a regular basis. From Figure 4 
we could deduce that two areas need a follow-up and a tailored social marketing plan: most of the Europeans, independent of their declared religious faith, are not very attracted to innovative products and services, and think that their advantages are often exaggerated. Perhaps this is the reason why the European Commission launched the Demand Side Policies for Innovation (https://ec.europa.eu/growth/industry/innovation/policy/demand-side-policies_en). If we could know the particularities felt by the different religious groups in Europe towards innovation and innovative products and services, and their evolution, then it would be possible to design a social marketing campaign to manage and improve the innovation perceived image among the Europeans. It would also help to balance the measures that historically have been deployed, almost exclusively, to the supply-side of the innovations.

As we have seen in Table 7, projections for 2050 did not seem to hold the secularization postulates in Europe (76.7\% of the Europeans would have a religion affiliation). While Christian Churches would still be predominant $(65.2 \%$ in 2050$)$ they would reduce their share in favor of Islamic creeds (from $5.9 \%$ in 2010 to $10.2 \%$ in 2050). Religions had different perceptions about innovation that have to be properly managed by companies and social and political organizations. It should be taken into account that, on innovation terms in Europe, religion is here to stay and religion matters.

Author Contributions: Conceptualization, A.R.-R., M.R.-M. and M.V.R.-G.; Methodology, A.R.-R., M.R.-M. and M.V.R.-G.; Literature Review, A.R.-R. and M.V.R.-G.; Software, A.R.-R.; Validation, A.R.-R. and M.V.R.-G.; Formal analysis, A.R.-R. and M.R.-M.; Investigation, A.R.-R.; Data curation, A.R.-R.; Writing the original draft, A.R.-R.; Review and editing the manuscript, M.R.-M.

Funding: This research received no external funding.

Conflicts of Interest: The authors declare no conflict of interest.

\section{References}

Abramitzky, Ran. 2008. The Limits of Equality: Insights from the Israeli Kibbutz. The Quarterly Journal of Economics 123: 1111-59. [CrossRef]

Assouad, Alexander, and K. Praveen Parboteeah. 2018. Religion and Innovation. A Country Institutional Approach. Journal of Management, Spirituality and Religion 15: 20-37. [CrossRef]

Audretsch, David Bruce, Werner Bönte, and Jagannadha Pawan Tamvada. 2007. Religion and Entrepeneurship. Paper Number 6378. Discussion Paper Series; London: CEPR.

Azzi, Corry, and Ronald Ehrenberg. 1975. Household Allocation of Time and Church Attendance. Journal of Political Economy 83: 27-56. [CrossRef]

Barro, Robert Joseph, and Rachel M. McCleary. 2003. Religion and Economic Growth across Countries. American Sociological Review 68: 760-81. [CrossRef]

Bartus, Tamás. 2005. Estimation of Marginal Effects Using Margeff. Stata Journal 5: 309-29. [CrossRef]

Basedau, Matthias, Simone Gobien, and Sebastian Prediger. 2018. The Multidimensional Effects of Religion on Socioeconomic Development: A Review of the Empirical Literature. Journal of Economic Surveys 32: 1106-33. [CrossRef]

Becker, Sascha O., and Ludger Woessmann. 2009. Was Weber Wrong? A Human Capital Theory oF Protestant Economic History. The Quarterly Jounal of Economics 124: 531-96. [CrossRef]

Bénabou, Roland, Davide Ticchi, and Andrea Vindigni. 2015. Moral Values and Economic Behavior: Religion and Innovation. American Economic Review: Papers \& Proceedings 105: 346-51. [CrossRef]

Bénabou, Roland, Davide Ticchi, and Andrea Vindigni. 2013. Forbidden Fruits: The Political Economy of Science, Religion, and Growth. NBER Working Paper Series 80. [CrossRef]

Bever, Edward, and Randall Styers. 2017. Magic in the Modern World: Strategies of Repression and Legitimization. Edited by Edward Bever and Randall Styers. Philadelphia: Pennsylvania State University Press.

Brooks, Harvey. 1994. The Relationship between Science and Technology. Research Policy 23: 477-86. [CrossRef]

Bruce, Steve. 1992. Religion and Modernization: Sociologists and Historians Debate the Secularization Thesis. Oxford \& New York: Oxford University Press.

Bruce, Steve. 2002. God Is Dead: Secularization in the West. Oxford: Wiley-Blackwell.

Bruce, Steve. 2011. Defining Religion: A Practical Response. International Review of Sociology 21: 107-20. [CrossRef] 
Bruce, Steve. 2013. Secularisation: In Defence of an Unfashionable Theory. Oxford: Oxford University Press.

Carpantier, Jean Francois, and Anastasia Litina. 2019. Dissecting the Act of God: An Exploration of the Effect of Religiosity on Economic Activity. B.E. Journal of Macroeconomics 19: 1-26. [CrossRef]

Cavalcanti, Tiago V., Stephen L. Parente, and Rui Zhao. 2007. Religion in Macroeconomics: A Quantitative Analysis of Weber's Thesis. Economic Theory 32: 105-23. [CrossRef]

Center for Religion Studies. Fondazione Bruno Kessler. 2018. Religion and Innovation. Calibrating Research Approaches and Suggesting Strategies for a Fruitful Interaction. Lavis: Esperia.

Cooper, Zaki, and Martha Patrick-Morsman. 2019. Why Many of Us Still Have Faith in Religion in This Scientific Age. The Guardian. Available online: https://www.theguardian.com/world/2019/jul/12/why-many-of-us-stillhave-faith-in-religion-in-this-scientific-age (accessed on 17 October 2019).

Crippen, Timothy. 1988. Old and New Gods in the Modern World: Toward a Theory of Religious Transformation. Social Forces 67: 316-36. [CrossRef]

Damm, Darlene, Rob Nail, and Adam Hofmann. 2019. The State of the Global Grand Challenges 2019. Edited by Merry Richter and Leslie O'Neill. Santa Clara: Singularity University.

Davie, Grace. 2002. Europe: The Exceptional Case. Parameters of Faith in the Modern World. London: Darton, Longman \& Todd.

Davis, Erik. 2017. Babalon Launching: Jack Parsons, Rocketry, and the 'Method of Science'. In Magic in the Modern World: Strategies of Repression and Legitimization. Edited by Edward Bever and Randall Styers. Philadelphia: The Pennsylvania University Press, pp. 119-46.

de Zwart, Frank. 2015. Unintended but Not Unanticipated Consequences. Theory and Society 44: 283-97. [CrossRef] Durkheim, Émile. 1925. Les Formes Élémentaires de La Vie Religieuse. Paris: Alcan.

Durkheim, Émile. 1926. De La Division Du Travail Social. Paris: Félix Alcan.

Eisenstadt, Shmuel Noah. 1968. The Protestant Ethic and Modernization: A Comparative View. New York: Basic Books.

European Commission. 2009. Responsible Research $\mathcal{E}$ Innovation. H2020. Available online: https://ec.europa.eu/ programmes/horizon2020/en/h2020-section/responsible-research-innovation (accessed on 17 October 2019).

European Commission. 2015. State of the Innovation Union 2015. Luxembourg: Publications Office of the European Union.

European Communities. 2005. Eurobarometer 63.4. Basic Bilingual Questionnaire Tns Opinion E Social. Luxembourg: European Communities.

Findlen, Paula, and Hannah Marcus. 2012. Science under Inquisition: Heresy and Knowledge in Catholic Reformation Rome. Isis 103: 376-82. [CrossRef]

Finke, Roger. 2013. Presidential Address: Origins and Consequences of Religious Freedoms: A Global Overview. Sociology of Religion: A Quarterly Review 74: 297-313. [CrossRef]

Finke, Roger, and Rodney Stark. 1988. Religious Economies and Sacred Canopies: Religious Mobilization in American Cities, 1906. American Sociological Review 53: 41. [CrossRef]

Gellner, Ernest. 1992. Postmodernism, Reason and Religion. London: Routledge.

Guiso, Luigi, Paola Sapienza, and Luigi Zingales. 2003. People's Opium? Religion and Economic Attitudes. Journal of Monetary Economics 50: 225-82. [CrossRef]

Hackett, Conrad, Brian J. Grim, Marcin Stonawski, Vegard Skirbekk, Michaela Potančoková, and Phillip Connor. 2012. The Global Religious Landscape. Washington, DC: Pew Research Center. [CrossRef]

Hair, Joseph F., William C. Black, Barry J. Babin, and Rolph E. Anderson. 2014. Multivariate Data Analysis, 7th ed. Essex: Pearson Education Limited.

Huang, Dan, Dong Lu, and Jin Hui Luo. 2016. Corporate Innovation and Innovation Efficiency: Does Religion Matter? Nankai Business Review International 7: 150-91. [CrossRef]

Hungerman, Daniel. 2005. Are Church and State Substitutes? Evidence from the 1996 Welfare Reform. Journal of Public Economics 89: 2245-67. [CrossRef]

Iannaccone, Laurence. 1992. Sacrifice and Stigma: Reducing Free-Riding in Cults, Communes, and Other Collectives. Journal of Political Economy 100: 271-91. [CrossRef]

Iannaccone, Laurence. 1998. Introduction to the Economics of Religion. Journal of Economic Literature 36: 1465-95. Iannaccone, Laurence. 2006. The Market for Martyrs. Interdisciplinary Journal of Research on Religion 2: 1-29.

Iyer, Sriya. 2016. The New Economics of Religion. Journal of Economic Literature 54: 395-441. [CrossRef]

Lazarus, Suleman. 2019. Where Is the Money? The Intersectionality of the Spirit World and the Acquisition of Wealth. Religions 10: 146. [CrossRef] 
Levy, Gilat, and Ronny Razin. 2012. Religious Beliefs, Religious Participation, and Cooperation. American Economic Journal Microeconomics 4: 121-51. [CrossRef]

Liu, Zhen, Qingke Guo, Peng Sun, Zhao Wang, and Rui Wu. 2018. Does Religion Hinder Creativity? A National Level Study on the Roles of Religiosity and Different Denominations. Frontiers in Psychology, 1-12. [CrossRef]

Manyika, James, Michael Chui, Jacques Bughin, Richard Dobbs, Peter Bisson, and Marrs. 2013. Disruptive Technologies: Advances That Will Transform Life, Business, and the Global Economy; McKinsey Global Insitute, May. Available online: http://www.mckinsey.com/insights/business_technology/disruptive_technologies\% 5Cnhttp://www.chrysalixevc.com/pdfs/mckinsey_may2013.pdf (accessed on 17 October 2019).

Marx, Karl. 1843. Critique of Hegel's Philosophy of Right. Cambridge: Cambridge University Press.

Marx, Karl, and N I Stone. 2014. A Contribution to the Critique of Political Economy. Chicago: Charles H. Kerr \& Company, (The Project Gutemberg EBook).

McBride, Michael. 2010. Religious Market Competition in a Richer World. Economica 77: 148-71. [CrossRef]

McCleary, Rachel M. 2011. The Economics of Religion as a Field of Inquiry. In THe Oxford Handbook of the Economics of Religion. New York: Oxford University Press.

McCleary, Rachel M., and Robert Joseph Barro. 2019. The Wealth of Religions. The Political Economy of Believing and Belonging. Princeton \& Oxford: Princeton University Press.

Michalopoulos, Stelios, Alireza Naghavi, and Giovanni Prarolo. 2016. Islam, Inequality and Pre-Industrial Comparative Development. Journal of Development Economics 120: 86-98. [CrossRef]

Milanovic, Branco. 2012. Globalization and Inequality. In The Globalization of the World Economy. Edited by Mark Casson. Cheltenham and Northampton: Edward Elgar Publishing Limited.

Montgomery, James Douglas. 2003. A Formalization and Test of the Religious Economies Model. American Sociological Review 68: 782. [CrossRef]

Mulgan, Geoff. 2016. Good and Bad Innovation: What Kind of Theory and Practice Do We Need to Distinguish Them? London: Nesta.

Niilola, Ian. 2017. Unanticipated and Undesirable Consequences of Technology in Management Research. Helsinki: Hanken School of Economics.

Niilola, Ian. 2018. Unanticipated and Undesirable Consequences of Innovation. Available online: https: //www.viima.com/blog/unanticipated-and-undesirable-consequences-of-innovation\#concepts (accessed on 17 October 2019).

Norman, Donald A. 1998. The invisible computer: Why Good Products Can Fail, the Personal Computer Is So Complex, and Information Appliances Are the Solution. Cambridge, MA: MIT Press.

Norris, Pippa, and Ronald Inglehart. 2011. Sacred and Secular: Religion and Politics Worldwide, 2nd ed. Cambridge: Cambridge University Press.

Noussair, Charles N, Stefan T. Trautmann, Gijs van de Kuilen, and Nathanael Vellekoop. 2013. Risk Aversion and Religion. Journal of Risk and Uncertainty. [CrossRef]

OECD. 2015. The Innovation Imperative: Contributing to Productivity, Growth and Well-Being. Paris: OECD Publishing.

Pals, D. L. 2006. Eight Theories of Religions. New York \& Oxford: Oxford University Press.

Parboteeah, K. Praveen, Sascha G. Walter, and Jörn H. Block. 2015. When Does Christian Religion Matter for Entrepreneurial Activity? The Contingent Effect of a Country's Investments into Knowledge. Journal of Business Ethics 130. [CrossRef]

Peifer, Jared L., David R. Johnson, and Elaine Howard Ecklund. 2019. The Moral Limits of the Market: Science Commercialization and Religious Traditions. Journal of Business Ethics 157: 183-97. [CrossRef]

Pew Research Center Religion and Public Life. 2015. The Future of World Religions: Population Growth Projections, 2010-2050. Washington, DC: Pew Research Center, Available online: http://www.pewforum.org/2015/04/02/ religious-projections-2010-2050/ (accessed on 17 October 2019).

Rogers, Everett M. 1995. Diffusion of Innovations. New York: The Free Press.

Rotman, David. 2013. How Technology Is Destroying Jobs. MIT Technology Review 116: 27-35.

Rubin, Jared. 2011. Institutions, the Rise of Commerce and the Persistence of Laws: Interest Restrictions in Islam and Christianity. Economic Journal 121: 1310-39. [CrossRef]

SAS. 2018. Computing Marginal Effects for Discrete Dependent Variable Models. Available online: https: //support.sas.com/rnd/app/ets/examples/margeff/index.html (accessed on 17 October 2019).

Sherkat, Darren E. 2017. Religion, Politics, and Americans' Confidence in Science. Politics and Religion 10: 137-60. [CrossRef] 
Simpson, J.A., E.S.C. Weiner, and Oxford University Press. 1989. The Oxford English Dictionary. Oxford: Clarendon Press.

Smith, Adam. 1776. An Inquiry Into the Nature and Causes of the Wealth of Nations. Edited by A. L. Macfie and D. D. Raphael. Indianapolis: Liberty Press.

Stark, Rodney. 1992. Do Catholic Societies Really Exist? Rationality and Society 4: 261-71. [CrossRef]

Stark, Rodney. 1999. Secularization, R.I.P. Sociology of Religion 60: 249-74. [CrossRef]

Stark, Rodney, Laurence R. Iannaccone, and Roger Finke. 1996. Religion, Science, and Rationality. The American Economic Review 86: 433-37.

The Economist. 2013. Has the Ideas Machine Broken-Down? The Economist, January 12.

The Economist. 2019. The Climate Issue -A Warming World. The Economist. Available online: https: //www.economist.com/leaders/2019/09/19/the-climate-issue. (accessed on 17 October 2019).

Weber, Max. 1930. The Protestant Ethic and the Spirit of Capitalism. London \& New York: Rouletge.

Weber, Max. 1946. Science as a Vocation. In From Max Weber: Essays in Sociology. Edited by H.H. Gerth and C. Wright Mills. New York: Oxford University Press, pp. 139-54.

Weber, Max. 1978. Economy and Society. Berkeley \& Los Angeles: University of California Press.

Yerxa, Donald A. 2016. Religion and Innovation. Antagonists or Partners? London \& New York: Bloomsbury Publishing.

Zielińska, Katarzyna. 2013. Concepts of Religion in Debates on Secularization. Approaching Religion 3: $25-35$. [CrossRef]

(C) 2019 by the authors. Licensee MDPI, Basel, Switzerland. This article is an open access article distributed under the terms and conditions of the Creative Commons Attribution (CC BY) license (http://creativecommons.org/licenses/by/4.0/). 